\title{
Chilling and Heat Requirements of Temperate Stone Fruit Trees (Prunus sp.)
}

\author{
Erica Fadón ${ }^{1, * \mathbb{C}}$, Sara Herrera ${ }^{2}$, Brenda I. Guerrero ${ }^{2}$, M. Engracia Guerra ${ }^{3}$ and \\ Javier Rodrigo $2,4, *$ (i)
}

1 INRES - Gartenbauwissenschaft, Universität Bonn, 53229 Bonn, Germany

2 Unidad de Hortofruticultura, Centro de Investigación y Tecnología Agroalimentaria de Aragón (CITA), Gobierno de Aragón, Avda. Montañana 930, 50059 Zaragoza, Spain; sherreral@aragon.es (S.H.); guerrero.bren@gmail.com (B.I.G.)

3 Departamento de Hortofruticultura, CICYTEX-Centro de Investigación 'Finca La Orden-Valdesequera', A-V, km 372, 06187 Guadajira, Badajoz, Spain; mariaengracia.guerra@juntaex.es

4 Instituto Agroalimentario de Aragón - IA2 (CITA-Universidad de Zaragoza), Calle Miguel Servet 177, 50013 Zaragoza, Spain

* Correspondence: jrodrigo@aragon.es (J.R); efadonad@uni-bonn.de (E.F.); Tel.: +34-976-716-314 (J.R); +49-(0)228-73-5155 (E.F.)

Received: 13 February 2020; Accepted: 16 March 2020; Published: 18 March 2020 updates

\begin{abstract}
Stone fruit trees of genus Prunus, like other temperate woody species, need to accumulate a cultivar-specific amount of chilling during endodormancy, and of heat during ecodormancy to flower properly in spring. Knowing the requirements of a cultivar can be critical in determining if it can be adapted to a particular area. Growers can use this information to anticipate the future performance of their orchards and the adaptation of new cultivars to their region. In this work, the available information on chilling- and heat-requirements of almond, apricot, plum, peach, and sweet cherry cultivars is reviewed. We pay special attention to the method used for the determination of breaking dormancy, the method used to quantify chilling and heat temperatures, and the place where experiments were conducted. The results reveal different gaps in the information available, both in the lack of information of cultivars with unknown requirements and in the methodologies used. The main emerging challenges are the standardization of the conditions of each methodology and the search for biological markers for dormancy. These will help to deal with the growing number of new cultivars and the reduction of winter cold in many areas due to global warming.
\end{abstract}

Keywords: almond; apricot; chilling hours; chilling units; chilling portions; European plum; growing degree hours; Japanese apricot; Japanese plum; peach; sour cherry; sweet cherry

\section{Introduction}

Temperate stone fruits belong to the genus Prunus in the Rosaceae and produce a fruit called drupe, whose seed is covered by the woody endocarp which in turn is covered by the endocarp. In most cultivated Prunus species, the edible part of the fruit is the endocarp, which includes the fleshy pulp (mesocarp) and skin (exocarp) such as apricot (P. armeniaca L.), European plum (P. domestica L.), Japanese apricot (P. mume Siebold and Zucc.), Japanese plum (P. salicina Lindl.), peach (P. persica L. Batsch), sour cherry (P. cerasus L.) and sweet cherry (P. avium L.) [1]. On the other hand, in almond (P. dulcis (Mill.) D.A. Webb), the edible part of the fruit is the seed. The annual global stone fruit production reached in 2017 more than 47 million $t$ in 7.3 million ha [2]. The most cultivated species are peach (P. persica L. Batsch) (24.6 million $\mathrm{t}$ in 1.5 million ha), plum (including European and Japanese plum) (11.7 million $\mathrm{t}$ in 2.6 million ha), apricot (4.2 million $\mathrm{t}$ in 0.5 million ha), sweet cherry 
(2.4 million $\mathrm{t}$ in 0.4 million ha), almond (2.2 million $\mathrm{t}$ in 1.9 million ha) and sour cherry (1.2 million $\mathrm{t}$ in 0.2 million ha) [2].

Stone fruit trees, like other temperate woody species, need to accumulate a cultivar-specific amount of chilling during winter to overcome dormancy and then experience warm temperatures to finally flower in spring [3-5]. These conditions the adaptation of species and cultivars to each region [6] and it is the main drawback for their extension to warmer latitudes [7]. Knowing the temperature requirements of a cultivar can be useful for growers to anticipate the future performance of their orchards and to design new orchards taking into account the predicted global warming [7-9]. In this work, the available information on chilling- and heat-requirements of cultivars of the most cultivated stone fruit crops (almond, apricot, peach, plum and cherry) is reviewed, paying special attention to the approach used for the determination of breaking dormancy, the method used to quantify chilling and heat temperatures, and the place where the experiments were conducted. There is extensive information available about chilling and heat requirements that has purposefully been omitted from this review. We have only included those studies that a) obtained results by using an experimental methodology (i.e., transferring shoots into a growth chamber sequentially during winter) or computational/statistical approaches that relate flowering dates to temperature data over a sufficiently long time series, and $b$ ) quantified chilling and heat temperatures using the common models (Chilling Hours model, Utah model or Dynamic model for chilling requirements, Growing Degree Hours for heat requirements).

\section{Dormancy: Definition and Description}

Stone fruit trees adapt to temperate regions by establishing a dormancy state during winter that allows surviving at low temperatures [10]. Dormancy characterizes by the absence of growth since flower primordia remain protected inside the buds. Growth is not only suppressed by the low temperatures since dormant trees do not respond to suitable conditions to grow and need exposure to a certain period of low temperatures to overcome dormancy [11]. It seems clear that dormancy is triggered by internal factors inherent to the plant [12]; however, up to now, most of these physiological factors remain unclear. The exposition to chilling temperatures allows a progressive restoration of growth capability. However, growth is not immediately restored [3], since low temperatures could continue and prevent buds from growing, and the exposure to warm temperatures is needed to grow after dormancy release [13]. Chilling and heat requirements are genetically determined and therefore are cultivar specific $[14,15]$.

The phases of chilling and warm temperature accumulation are differently named, although they occur at the same phenological stage [16]. Lang et al. proposed one of the most used terms, naming the stages "endo-dormancy" while chilling accumulates, and "eco-dormancy" while heat accumulates and chilling prevents the plant from growing [11]. On the other hand, a recent proposal by Considine and Considine considered that dormancy only refers to when it is internally caused, and the lack of growth by external factors correspond to a quiescence state [17].

Dormancy has acquiring rising importance for a sustainable fruit production under a global warming context $[8,9,18-20]$. However, the physiological processes behind remain unknown and a reliable biological factor linked to the dormancy breaking is still missing [12].

\section{Dormancy prediction}

The characterization of the temperature requirements of a cultivar is crucial for the design and management of the fruit orchards since they determine the flowering date and a flowering overlap is needed for cross-pollination in case of self-incompatible cultivars [21]. It is also a key trait in breeding programs since it determines both the adaptation to different climates and the blooming and ripening dates [22]. However, the lack of a whole understanding of the process makes obtaining reliable data complex [6]. In most works, the determination of the temperature requirements of a particular cultivar consists of two phases (Figure 1): first, the establishment of the dormancy and forcing periods 
(Figure 1a), either experimentally (Figure 1a.1) [23,24] or statistically (Figure 1a.2) [25-27]; and the subsequent temperature quantification in both phases (Figure 1b) by using temperature-based models for chilling (Figure 1b.1) [28-30] and heat quantification (Figure 1b.2) [29]. One of the main challenges of determining the temperature requirements is establishing the transition from dormancy to ecodormancy (Figure 1a) and thus the periods in which chilling (Figure 1b.1) and warm temperatures (Figure 1b.2) are quantified.

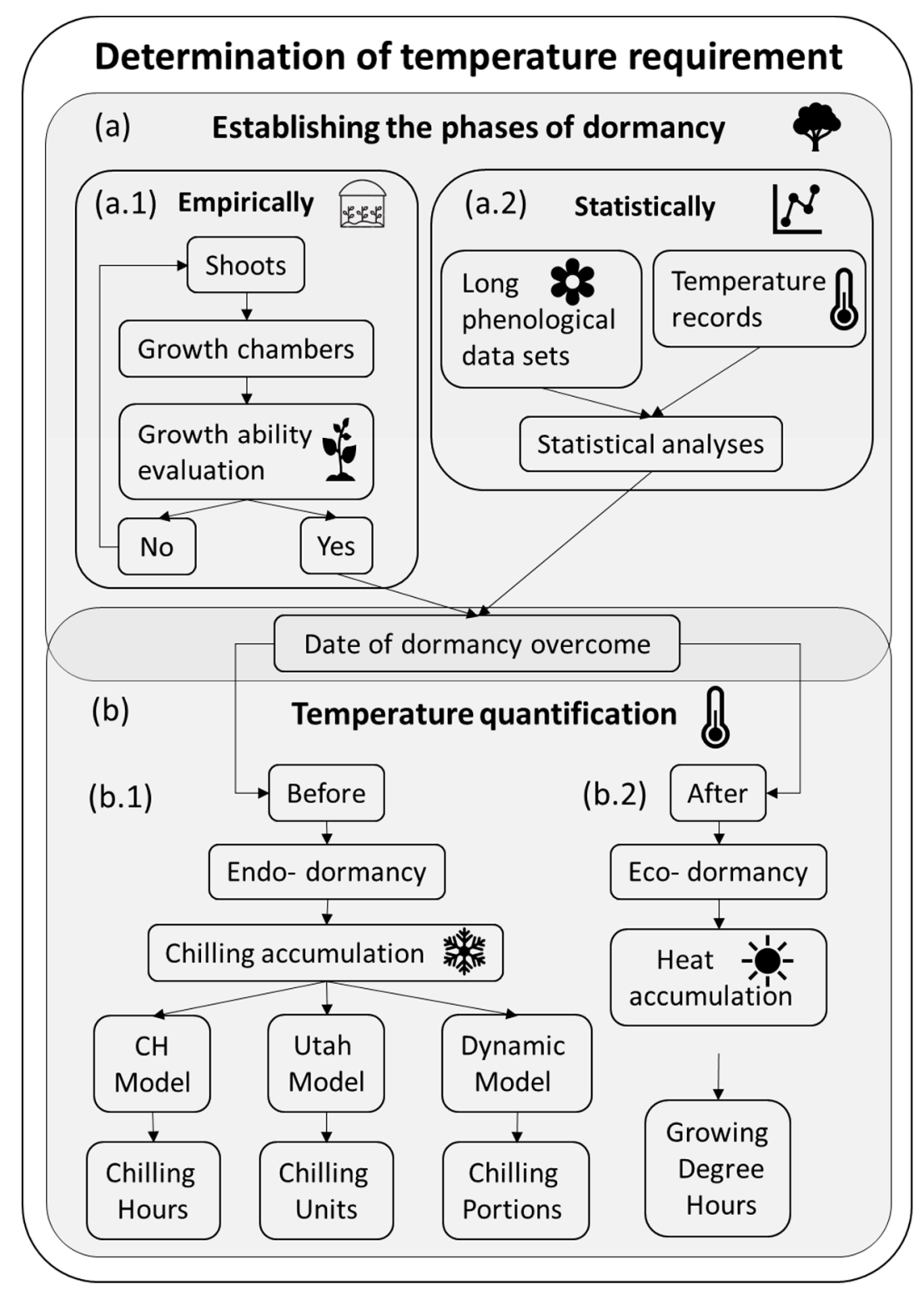

Figure 1. Determination of the temperature (chill and heat) requirements in temperate fruit trees: a workflow. (a) Determination of the phases of dormancy: (a.1) empirically or (a.2) statistically. (b) Temperature quantification: (b.1) chilling quantification during endo-dormancy and (b.2) heat quantification over eco-dormancy.

The experimental determination of dormancy consists of evaluating when the buds recover the capacity to grow (Figure 1a.1). This is usually performed by transferring shoots into a growth chamber sequentially during winter, thus after different chilling exposures. Shoots remain a certain period in the warm conditions, and then bud growth is evaluated. This approach has been widely used from 
early [24] to recent studies [31] that determine the chilling requirements of the cultivars. Furthermore, these experiments serve as a base for physiological studies on dormancy [6].

The statistical approach estimates the date of chilling fulfillment based on a long series of phenological observations (flowering dates) and relating them with the previous temperature records (Figure 1a.2). Tabuenca et al. established a statistical methodology by calculating the correlation coefficients between the maximum, minimum and mean temperatures of certain time periods and flowering dates in apple, apricot, cherry, peach, pear, plum [32], and almonds [33]. Then, Alonso et al. determined the temperature requirements correlations between the flowering dates of almond cultivars and daily minimum, mean and maximum temperatures calculated as the mean of the surrounding 5 , $10,15 \ldots$ until 30 days, with a set of data from 7 years. The endo-dormancy to eco-dormancy transition was considered to be when the significant correlation coefficients change from being mainly positive to be mainly negative [26]. Ashcroft et al. firstly estimated chilling and heat accumulation of peaches [29] based on when the chilling and heat accumulation presented the least squared residuals methods [25]. A new approach has been recently developed based on the statistical analysis of long-term phenological records and temperature series. The application of partial least squares (PLS) regression leads to the estimation of the agroclimatic requirements. PLS regression is especially applicable when the number of independent variables (daily temperatures, 365 data per year) substantially exceeds the number of dependent variables (one flowering date per cultivar and year). The results of these analyses include the model coefficients and variable-importance-in-the-projection. Significant positive model coefficients correspond with the chilling accumulation, endo- dormancy, while negative coefficients correspond with the heat accumulation, eco- dormancy [34]. It was initially applied in sweet cherry [34] and later in other fruit trees as almond [35,36], pistachio [19], apricot [27] or apple [36].

\section{Temperature Based Models for Phenology Prediction}

Three main models are currently used in agriculture to quantify chilling over the dormancy period [37]. They were developed in peach: the Chilling Hours model [28], the Utah model [29], and the Dynamic model [30] (Figure 1b.1). The Chilling Hours model was developed in the early fifties of the $20^{\text {th }}$ century, and it has been widely used up to now due to its simplicity and easy comprehension and calculation. This model establishes that a Chilling Hour $(\mathrm{CH})$ corresponds to an hour at temperature between 0 and $7.2^{\circ} \mathrm{C}\left(45^{\circ} \mathrm{F}\right)$, since this range of temperatures is considered to affect dormancy completion. While temperatures below $0{ }^{\circ} \mathrm{C}$ are assumed not contributing due to at such low temperatures biological processes were considered slowed or not occurring, temperatures over $7.2{ }^{\circ} \mathrm{C}(45 \mathrm{~F})$ were considered not low enough to affect dormancy completion [28].

The Utah model bases on the quantification of Chilling Units (CU) and establishes different ranges of temperatures with a different contribution to dormancy completion. A chilling unit corresponds to one hour under temperatures between $2.5-9.1{ }^{\circ} \mathrm{C}$, a range that is considered the most effective temperatures on dormancy completion. Other ranges of temperatures are considered to have half $\left(1.5-2.4{ }^{\circ} \mathrm{C}\right.$ and $\left.9.2-12.4{ }^{\circ} \mathrm{C}\right)$, null $\left(<1.4{ }^{\circ} \mathrm{C}\right.$ and $\left.12.5-15.9{ }^{\circ} \mathrm{C}\right)$ or negative $\left(>16^{\circ} \mathrm{C}\right)$ contribution to dormancy [29].

The Dynamic model, dated back from the 1980s [30], is based on a series of experiments that evaluated the effect of different series of temperatures on dormancy release [38-40]. This model proposed the accumulation of an intermediate product promoted by cold temperatures that can be reversed by warm temperatures (first step). Once this intermediate product has reached a certain level, the chill portions are permanently fixed and are considered not affected by warm temperatures [30]. The model is based on a possible biological process in which a thermally unstable precursor would lead to the accumulation of a factor in the buds. This process would follow the Arrhenius law that fits the mathematical relationship between temperature and the rate of a chemical reaction.

Once dormancy is predicted allowing the quantification of chilling, it is also needed to quantified warm temperatures after dormancy for flowering to occur (Figure 1b.2). The modelization of warm temperatures was early developed in agriculture to predict the different phenological stages of crops [41]. 
The combination of a chilling model with a heat model to predict flowering was firstly described with the combination of the Utah model with the Growing Degree Hours (GDH) quantification [25], and then this combination was also applied with the other chilling models. A GDH is defined as one hour at $1{ }^{\circ} \mathrm{C}$ above the base temperature $\left(4.5^{\circ} \mathrm{C}\right)$, this linearly progresses until the upper limit $\left(25^{\circ} \mathrm{C}\right)[13]$. One of the main drawbacks of using these models is the necessity of hourly temperature data records, whose availability is limited. Thus, equivalent models have been developed based on maximum and minimum temperatures [42].

\section{Chilling and Heat Requirements}

\subsection{Almond (P. dulcis)}

Although North America is the main area for almond production (over 1 million $t / y e a r)$ [2], temperature requirements have been calculated in the Mediterranean area, in Spain [26,33,36,43], the second world producer (0.6 million t/year) [2] and Tunisia [35]. Temperature requirements (chilling and heat) are available for a total of 106 almond cultivars [26,33,35,36,43] (Table 1). The chilling requirements varies between 'Achaak' (8 CH/ -297CU/3.4CP) [35] and 'R1000' (996 CU) [43]. The heat requirements range from 2894 GDH for 'Pizzuta' to 10201 GDH for 'Primorskiy' [36].

In this species, the most data (96 out of 106 cultivars) were calculated with statistical approaches, which contrast with the other Prunus sp. reported in this work. Almond data were obtained according to three different statistical methodologies [26,33,34]. The initial phenological data set also differed between works: the PLS analysis was performed over the date of flowering initiation (BBCH phenological stage 61, 10\% flowers open) during 30 years [34,35], while the other approaches based on the dates of full bloom (BBCH phenological stage 65, 50\% flowers open) over 7 [26] and 4-10 years [33].

A comparison between experimental (E) [43] and statistical (S) [26] approaches reveals similar results of chilling requirements and heat requirements for 'Ferragnès' (558 and 444 CU, 7309 and 8051 GDH), 'Marcona' (435 and 428 CU, 6681 and 6603 GDH) and 'Ramillete' (326 and 444 CU, 6538 and 5947 GDH) in Spain. Unfortunately, it is not possible to make more comparisons due to the different models used to quantify chilling and the different cultivars used in each study.

Table 1. Chilling and heat requirements of almond cultivars.

\begin{tabular}{ccccccccc}
\hline \multirow{2}{*}{ Cultivar } & \multicolumn{2}{c}{ Chilling requirements } & Heat requirements & \multirow{2}{*}{ Method } & Loc. & \multirow{2}{*}{ Ref. } \\
\hline A-258 & - & - & $17 \pm 4.5$ & $8725 \pm 1712$ & S & Spain & {$[36]$} \\
\hline Abiodh de Sfax & 12 & -284 & 4.6 & 6206 & S & Tunisia & {$[35]$} \\
\hline Abiodh Ras Djebel & 59 & -53 & 15.5 & 7324 & S & Tunisia & {$[35]$} \\
\hline \multirow{2}{*}{ Achaak } & - & 266 & - & 6444 & E & Spain & {$[43]$} \\
\cline { 2 - 9 } & 8 & -297 & 3.4 & 8703 & S & Tunisia & {$[35]$} \\
\hline \multirow{2}{*}{ Aï } & - & 444 & - & 8051 & S & Spain & {$[26]$} \\
\hline Alicante & 169 & - & - & Very high & S & Spain & {$[33]$} \\
\hline \multirow{2}{*}{ Alzina } & - & - & $21.7 \pm 4.7$ & $6940 \pm 1400$ & S & Spain & {$[36]$} \\
\hline Amargo & - & 463 & - & 6757 & S & Spain & {$[26]$} \\
\hline Andreu & 408 & - & - & Very low & S & Spain & {$[33]$} \\
\hline Antoñeta & 169 & - & - & Low & S & Spain & {$[33]$} \\
\hline Ardechoise & 151 & - & - & - & High & S & Spain & {$[33]$} \\
\hline
\end{tabular}


Table 1. Cont.

\begin{tabular}{|c|c|c|c|c|c|c|c|}
\hline \multirow{2}{*}{ Cultivar } & \multicolumn{3}{|c|}{ Chilling requirements } & \multirow{2}{*}{$\begin{array}{c}\text { Heat requirements } \\
\text { GDH }\end{array}$} & \multirow{2}{*}{ Method } & \multirow{2}{*}{ Loc. } & \multirow{2}{*}{ Ref } \\
\hline & $\mathrm{CH}$ & $\mathrm{CU}$ & $\mathrm{CP}$ & & & & \\
\hline Avola & 50 & 46 & 13.6 & 6673 & $S$ & Tunisia & [35] \\
\hline Aylés & - & 481 & - & 7909 & $S$ & Spain & [26] \\
\hline Bertina & - & 463 & - & 8536 & $S$ & Spain & [26] \\
\hline Blanquerna & - & 463 & - & 6906 & $S$ & Spain & [26] \\
\hline Bonifacio & 61 & 101 & 15.8 & 7559 & $S$ & Tunisia & [35] \\
\hline Bruantine & 34 & -219 & 10.4 & 8548 & $S$ & Tunisia & [35] \\
\hline Cambra & - & 463 & - & 7697 & $S$ & Spain & [26] \\
\hline \multirow{2}{*}{ Cavaliera } & 34 & -219 & 10.4 & 7042 & $S$ & Tunisia & [35] \\
\hline & - & - & $11.6 \pm 4.0$ & $7452 \pm 1601$ & $S$ & Spain & {$[36]$} \\
\hline Chellastone & - & 463 & - & 6168 & $S$ & Spain & [26] \\
\hline Chine & 151 & - & - & Low & $S$ & Spain & [33] \\
\hline Constantini & - & 444 & - & 5345 & $S$ & Spain & [26] \\
\hline \multirow{3}{*}{ Cristomorto } & 83 & -29 & 22.6 & 5872 & $S$ & Tunisia & [35] \\
\hline & - & 428 & - & 8027 & $S$ & Spain & [26] \\
\hline & - & - & $20.7 \pm 4.7$ & $8236 \pm 1482$ & $S$ & Spain & {$[36]$} \\
\hline \multirow{2}{*}{ Desmayo } & - & 309 & - & 5942 & E & Spain & [43] \\
\hline & 169 & - & - & Medium & S & Spain & [33] \\
\hline \multirow{2}{*}{ Desmayo Largueta } & - & 428 & - & 5458 & $S$ & Spain & [26] \\
\hline & - & - & $8.4 \pm 3.7$ & $8552 \pm 1741$ & $S$ & Spain & {$[36]$} \\
\hline \multirow{2}{*}{ Desmayo Rojo } & - & 463 & - & 6418 & $S$ & Spain & [26] \\
\hline & 169 & - & - & High & $S$ & Spain & [33] \\
\hline Dorée & 46 & -174 & 12.7 & 8867 & $S$ & Tunisia & [35] \\
\hline Drake & 169 & - & - & Very high & $S$ & Spain & [33] \\
\hline Durán & 151 & - & - & Medium & $S$ & Spain & [33] \\
\hline Faggoussi & 54 & -148 & 14.5 & 3962 & $S$ & Tunisia & [35] \\
\hline Fakhfekh & 33 & -219 & 10.4 & 5979 & $S$ & Tunisia & [35] \\
\hline Fasciuneddu & 34 & -219 & 10.4 & 7027 & $S$ & Tunisia & [35] \\
\hline Felisia & - & 428 & - & 9352 & $S$ & Spain & [26] \\
\hline \multirow{2}{*}{ Ferraduel } & 54 & 59 & 14.4 & 9272 & S & Tunisia & [35] \\
\hline & - & - & $52.9 \pm 6.0$ & $7285 \pm 1362$ & S & Spain & [36] \\
\hline \multirow{4}{*}{ Ferragnès } & - & 558 & - & 7309 & $\mathrm{E}$ & Spain & [43] \\
\hline & 54 & 59 & 14.4 & 9215 & $S$ & Tunisia & [35] \\
\hline & - & 444 & - & 8051 & $S$ & Spain & [26] \\
\hline & - & - & $20.7 \pm 4.7$ & $8696 \pm 1543$ & $S$ & Spain & [36] \\
\hline \multirow{2}{*}{ Filippo Ceo } & - & 463 & - & 7558 & $S$ & Spain & [26] \\
\hline & 483 & - & - & Low & $S$ & Spain & [33] \\
\hline Fourcouronne & 169 & - & - & High & $S$ & Spain & [33] \\
\hline \multirow{3}{*}{$\begin{array}{l}\text { Fournat de } \\
\text { Brézenaud }\end{array}$} & 169 & - & - & Very high & $\mathrm{S}$ & Spain & [33] \\
\hline & - & 416 & - & 7367 & $S$ & Spain & [26] \\
\hline & 79 & -50 & 21.1 & 5368 & $S$ & Tunisia & [35] \\
\hline Gabaix & - & - & $13.4 \pm 4.4$ & $6824 \pm 1421$ & $S$ & Spain & [36] \\
\hline Garbí & - & - & $52.9 \pm 6$ & $7040 \pm 1312$ & $S$ & Spain & [36] \\
\hline Garnghzel & 12 & -284 & 4.6 & 8703 & $S$ & Tunisia & [35] \\
\hline
\end{tabular}


Table 1. Cont.

\begin{tabular}{|c|c|c|c|c|c|c|c|}
\hline \multirow{2}{*}{ Cultivar } & \multicolumn{3}{|c|}{ Chilling requirements } & \multirow{2}{*}{$\begin{array}{c}\text { Heat requirements } \\
\text { GDH }\end{array}$} & \multirow{2}{*}{ Method } & \multirow{2}{*}{ Loc. } & \multirow{2}{*}{ Ref } \\
\hline & $\mathrm{CH}$ & $\mathrm{CU}$ & $\mathrm{CP}$ & & & & \\
\hline Garrigues & - & - & $22.0 \pm 4.7$ & $8054 \pm 1811$ & $S$ & Spain & {$[36]$} \\
\hline \multirow{2}{*}{ Genco Taronto } & 80 & 194 & 21.4 & 6148 & $S$ & Tunisia & [35] \\
\hline & - & - & $28.7 \pm 4.9$ & $5971 \pm 1189$ & $S$ & Spain & {$[36]$} \\
\hline Glorieta & - & - & $51.6 \pm 5.9$ & $5654 \pm 1177$ & $S$ & Spain & {$[36]$} \\
\hline Guara & - & 463 & - & 7978 & $S$ & Spain & [26] \\
\hline \multirow{2}{*}{ Jordi } & - & 428 & - & 6488 & $S$ & Spain & [26] \\
\hline & 151 & - & - & Medium & $S$ & Spain & [33] \\
\hline Khoukhi & 31 & -227 & 9.9 & 8873 & $S$ & Tunisia & [35] \\
\hline Ksontini & 21 & -258 & 7.3 & 7071 & $S$ & Tunisia & [35] \\
\hline Languedoc & 23 & -174 & 7.7 & 9097 & $S$ & Tunisia & [35] \\
\hline Lauranne & - & 428 & - & 8569 & $S$ & Spain & [26] \\
\hline LeGrand & - & 428 & - & 8027 & $S$ & Spain & [26] \\
\hline Lluch & & - & & Very high & $S$ & Spain & [33] \\
\hline Malagueña & 23 & -82 & 7.6 & 9224 & $\mathrm{~S}$ & Tunisia & [35] \\
\hline \multirow{4}{*}{ Marcona } & 169 & - & - & High & $S$ & Spain & [33] \\
\hline & - & 435 & - & 6681 & $\mathrm{E}$ & Spain & [43] \\
\hline & - & 428 & - & 6603 & $S$ & Spain & [26] \\
\hline & - & - & $22.0 \pm 4.7$ & $6378 \pm 1341$ & $S$ & Spain & [36] \\
\hline Marta & - & 478 & - & 7577 & $\mathrm{E}$ & Spain & [43] \\
\hline \multirow{2}{*}{ Masbovera } & - & 463 & - & 7841 & $S$ & Spain & [26] \\
\hline & - & - & $28.6 \pm 4.9$ & $6232 \pm 1221$ & $S$ & Spain & [36] \\
\hline Mazzetto & 54 & -68 & 14.5 & 9507 & $S$ & Tunisia & [35] \\
\hline \multirow{2}{*}{ Miagkoskorlupij } & - & 463 & - & 7439 & $S$ & Spain & [26] \\
\hline & 631 & - & - & Very low & $S$ & Spain & [33] \\
\hline Mollar de Tarragona & - & - & $20.0 \pm 4.7$ & $6718 \pm 1378$ & $S$ & Spain & {$[36]$} \\
\hline Moncayo & - & 463 & - & 8696 & $S$ & Spain & [26] \\
\hline Montrone & 31 & -227 & 9.9 & 9694 & $S$ & Tunisia & [35] \\
\hline Morskoi & 233 & - & - & Very high & $S$ & Spain & [33] \\
\hline \multirow{3}{*}{ Ne Plus Ultra } & 169 & - & - & Medium & $S$ & Spain & [33] \\
\hline & 50 & 11 & 13.6 & 6847 & $S$ & Tunisia & [35] \\
\hline & - & 463 & - & 6635 & $S$ & Spain & [26] \\
\hline \multirow{4}{*}{ Nonpareil } & 83 & -29 & 22.6 & 6045 & $S$ & Tunisia & [35] \\
\hline & - & 403 & - & 7758 & $S$ & Spain & [26] \\
\hline & 169 & - & - & High & $S$ & Spain & [33] \\
\hline & - & - & $21.7 \pm 4.7$ & $7062 \pm 1399$ & $S$ & Spain & [36] \\
\hline \multirow{2}{*}{ Picantilli } & - & 428 & - & 7386 & $S$ & Spain & [26] \\
\hline & 561 & - & - & Very low & $S$ & Spain & [33] \\
\hline Pizzuta & 83 & -29 & 22.6 & 2894 & $S$ & Tunisia & [35] \\
\hline \multirow{2}{*}{ Ponç } & - & 428 & - & 6210 & $S$ & Spain & [26] \\
\hline & 101 & - & - & High & $S$ & Spain & [33] \\
\hline Poleta & 151 & - & - & High & $S$ & Spain & [33] \\
\hline \multirow{2}{*}{ Pou de Felanitz } & - & 392 & - & 5419 & $S$ & Spain & [26] \\
\hline & 101 & - & - & Medium & $S$ & Spain & [33] \\
\hline
\end{tabular}


Table 1. Cont.

\begin{tabular}{|c|c|c|c|c|c|c|c|}
\hline \multirow{2}{*}{ Cultivar } & \multicolumn{3}{|c|}{ Chilling requirements } & \multirow{2}{*}{$\begin{array}{c}\text { Heat requirements } \\
\text { GDH }\end{array}$} & \multirow{2}{*}{ Method } & \multirow{2}{*}{ Loc. } & \multirow{2}{*}{ Ref } \\
\hline & $\mathrm{CH}$ & $\mathrm{CU}$ & CP & & & & \\
\hline Princesa & 169 & - & - & High & $S$ & Spain & [33] \\
\hline \multirow[b]{2}{*}{ Primorskij } & - & 428 & - & 8434 & $S$ & Spain & [26] \\
\hline & - & - & $\begin{array}{l}52.85 \pm \\
5.95\end{array}$ & $10201 \pm 1834$ & S & Spain & {$[36]$} \\
\hline R1000 & - & 996 & - & 7438 & E & Spain & [43] \\
\hline \multirow{3}{*}{ Rachele } & 233 & - & - & Very high & $S$ & Spain & [33] \\
\hline & - & 376 & - & 8302 & $S$ & Spain & [26] \\
\hline & 47 & -167 & 13.3 & 6374 & $S$ & Tunisia & [35] \\
\hline \multirow{3}{*}{ Ramillete } & - & 444 & - & 5947 & S & Spain & [26] \\
\hline & - & 326 & - & 6538 & $\mathrm{E}$ & Spain & [43] \\
\hline & - & - & $20.7 \pm 4.7$ & $6998 \pm 1540$ & $S$ & Spain & [36] \\
\hline Ramlet R249 & 33 & 18 & 10.3 & 6812 & $S$ & Tunisia & [35] \\
\hline Ramlet R250 & 19 & -266 & 6.7 & 6812 & $\mathrm{~S}$ & Tunisia & [35] \\
\hline Rana & - & - & $20.0 \pm 4.7$ & $6518 \pm 1292$ & $S$ & Spain & [36] \\
\hline \multirow{3}{*}{ Rof } & - & 463 & - & 6418 & $S$ & Spain & [26] \\
\hline & 169 & - & - & High & $S$ & Spain & [33] \\
\hline & - & - & $20.8 \pm 4.7$ & $6965 \pm 1355$ & S & Spain & [36] \\
\hline Rotjet & 151 & - & - & Low & S & Spain & [33] \\
\hline $\mathrm{S} 2332$ & - & 417 & - & 6481 & E & Spain & [43] \\
\hline S5133 & - & 973 & - & 7003 & E & Spain & [43] \\
\hline Sicilia & 151 & - & - & High & $\mathrm{S}$ & Spain & [33] \\
\hline Soukaret & 77 & -57 & 20.6 & 5960 & S & Tunisia & [35] \\
\hline $\begin{array}{l}\text { Tardive de la } \\
\text { Verdière }\end{array}$ & - & 358 & - & 8814 & S & Spain & [26] \\
\hline Tardy Nonpareil & - & - & $55.4 \pm 5.9$ & $9444 \pm 1658$ & S & Spain & [36] \\
\hline Tarragona & 83 & -29 & 22.6 & 5830 & $\mathrm{~S}$ & Tunisia & [35] \\
\hline Tarragones & - & - & $51.6 \pm 5.9$ & $6370 \pm 1238$ & S & Spain & [36] \\
\hline Tamarite 2 & 169 & - & - & High & S & Spain & [33] \\
\hline \multirow{3}{*}{ Texas } & - & 463 & - & 7697 & $S$ & Spain & [26] \\
\hline & 233 & - & - & High & $S$ & Spain & [33] \\
\hline & - & - & $51.6 \pm 5.9$ & $6280 \pm 1225$ & $\mathrm{~S}$ & Spain & [36] \\
\hline Thompson & - & 463 & - & 7697 & $S$ & Spain & [26] \\
\hline Titan & - & 444 & - & 8457 & $S$ & Spain & [26] \\
\hline Tokyo & - & 463 & - & 7558 & $S$ & Spain & [26] \\
\hline \multirow{2}{*}{ Totsol } & - & 428 & - & 6943 & $S$ & Spain & [26] \\
\hline & 101 & - & - & High & $S$ & Spain & [33] \\
\hline Tozeur 1 & 12 & -284 & 4.6 & 8124 & S & Tunisia & [35] \\
\hline Tozeur 2 & 33 & -219 & 10.4 & 6309 & S & Tunisia & [35] \\
\hline Tozeur 4 & 12 & -284 & 4.6 & 6698 & S & Tunisia & [35] \\
\hline \multirow{2}{*}{ Trell } & 77 & -57 & 20.6 & 6003 & $\mathrm{~S}$ & Tunisia & [35] \\
\hline & 233 & - & - & Medium & $S$ & Spain & [33] \\
\hline \multirow{3}{*}{ Tuono Taronto } & - & 463 & - & 7978 & $\mathrm{~S}$ & Spain & [26] \\
\hline & 50 & 46 & 13.6 & 6148 & $S$ & Tunisia & [35] \\
\hline & - & - & $52.9 \pm 6.0$ & $6870 \pm 1319$ & $S$ & Spain & [36] \\
\hline
\end{tabular}


Table 1. Cont.

\begin{tabular}{|c|c|c|c|c|c|c|c|}
\hline \multirow{2}{*}{ Cultivar } & \multicolumn{3}{|c|}{ Chilling requirements } & \multirow{2}{*}{$\begin{array}{c}\text { Heat requirements } \\
\text { GDH }\end{array}$} & \multirow{2}{*}{ Method } & \multirow{2}{*}{ Loc. } & \multirow{2}{*}{ Ref } \\
\hline & $\mathrm{CH}$ & $\mathrm{CU}$ & $\mathrm{CP}$ & & & & \\
\hline \multirow{2}{*}{ Verdereta } & - & 416 & - & 6606 & $S$ & Spain & [26] \\
\hline & 101 & - & - & High & S & Spain & [33] \\
\hline Verdiere & 169 & - & - & Very high & S & Spain & [33] \\
\hline Vinagrilla & 408 & - & - & Very low & S & Spain & [33] \\
\hline \multirow{2}{*}{ Vivot } & - & 428 & - & 6603 & $S$ & Spain & [26] \\
\hline & 151 & - & - & High & $S$ & Spain & [33] \\
\hline Xina & - & 403 & - & 5815 & S & Spain & [26] \\
\hline Yaltano & 713 & - & - & Very low & $S$ & Spain & [33] \\
\hline Yaltinskij & - & 463 & - & 8536 & $S$ & Spain & {$[26]$} \\
\hline \multirow{2}{*}{ Zahaf } & 31 & -227 & 9.9 & 6279 & $S$ & Tunisia & [35] \\
\hline & - & 392 & - & 5611 & $S$ & Spain & [26] \\
\hline
\end{tabular}

\subsection{European and Japanese Apricot (P. armeniaca and P. mume)}

European apricot is one of the most economically important fruit crops in temperate regions worldwide [44]. It is mainly produced in the Mediterranean area and the Middle East, being the higher producers Turkey, Uzbekistan, Italy, Algeria, and Iran [2]. A total of 15 works have experimentally evaluated the chilling requirements of 68 apricot cultivars all around the world (Iran, Italy, Serbia, South Africa, Spain, and the USA) (Table 2). The range of chilling requirements is between $274 \mathrm{CU}$ in 'Palsteyn' [45] to 1450-1600 CU in 'Orangered' [46]. This crop is cultivated mainly in Mediterranean regions and it has traditionally been considered that most cultivars had low chilling requirements. However, some traditional cultivars showed high chilling requirements as 'Búlida' (1048 CU), 'Canino' (806 CU), 'Currot' (642 CU) or 'Moniqui' (1139 CU) [42,43] (Table 2).

In the last decades, an important renewal is taking place due to sharka, a disease caused by the Plum Pox Virus (PPV). High chilling PPV-resistant cultivars from North America, such as 'Goldrich' (950-108 CU / 65-59 CP), 'Harcot' (920-1665 CP), 'Orangered' (568-1481 CH / 902-1600CU / 55-69 CP), and 'Stark Early Orange' (1411 CU / 79 CP) (Table 2), have been used as parentals in different breeding programs with the aim of introducing a source of the resistance to the disease. The release of a high number of new cultivars is resulting in a lack of information about the chilling and heat requirements of the majority of the new commercial cultivars $[44,47]$.

Some cultivars such as 'Aurora', 'Bergeron', 'Currot', 'Dorada', 'Goldrich', 'Laycot', 'Luizet', 'Moniqui', 'Murciana', 'Paviot', 'Rojo Pasión', 'Royal', 'San Castrese', and 'Selene' show homogeneous results in the different studies (Table 2). However, highly variable results have been reported in other cultivars as 'Cafona', 'Canino', 'Harcot', 'Orangered', 'Palsteyn', 'Polonais', 'Precoz de Colomber', and 'Tonda di Costiglione', showing heterogeneity among the different approaches (Table 2). Likewise, high differences have been reported when the experiments were carried out in different locations $[45,48,49]$ or years [48,50,51]. Even when the same cultivars ('Canino', 'Orangered' and 'Palsteyn') were evaluated using the same approach in two environments with different climatic conditions, the results obtained showed high differences, with higher values in Spain (806/1172/631 CU) than in South Africa (304/957/274 CU) [45] (Table 2). Heat requirements ranged from $485 \mathrm{GDH}$ in 'Goldrich' [49] to values above 6000 GDH in 'Canino' [48], 'Dorada', 'Palsteyn', and 'Rojo Pasión' [45]. Some cultivars showed high differences between seasons, as 'Cafona' (2499-5800 GDH), 'Canino' (2547-6729 GDH), and 'Precoz de Colomber' (2320-5304 GDH) [48].

Japanese apricot originated in China and has been widely cultivated for about 3000 years in Asian countries as China, Japan, and Korea. However, this crop is hardly known in other countries probably due to its poor adaptation to other areas of different climatic conditions, since it requires warmer and more humid conditions than European apricot [44]. 
Chilling requirements range from $26 \pm 7 \mathrm{CP}$ for 'Shuangshuidaroumei' [52] to $78.5 \mathrm{CP}$ for 'Sichuangqingmei' and 'Tengwulang' [53] (Table 3). Heat requirements vary from 822 GDH for 'Dayu' to 2378 GDH for 'Jietianmei', 'Sichuangqingmei', and 'Tengwulang' (Table 3) [53]. Japanese apricot shows high chilling requirements and extremely low heat requirements when compared with the other Prunus sp. reported in this study.

Some cultivars with a wide range of chilling requirements, such as 'Nanko', a high-chilling cultivar from Japan, and 'Ellching', a low-chilling cultivar from the subtropical region in Taiwan, have been used in studies on dormancy physiology [54] and genetic regulation [55-59].

Table 2. Chilling and heat requirements of European apricot cultivars.

\begin{tabular}{|c|c|c|c|c|c|c|c|}
\hline \multirow{2}{*}{ Cultivar } & \multicolumn{3}{|c|}{ Chilling requirements } & \multirow{2}{*}{$\begin{array}{c}\text { Heat requirements } \\
\text { GDH }\end{array}$} & \multirow{2}{*}{ Method } & \multirow{2}{*}{ Loc. } & \multirow{2}{*}{ Ref } \\
\hline & $\mathbf{C H}$ & $\mathrm{CU}$ & $\mathrm{CP}$ & & & & \\
\hline Abricot Pêche 1708 A.D. & $1015-1105$ & - & - & - & $\mathrm{E}$ & Spain & [60] \\
\hline Alessandrino & - & $1000-1140$ & - & 3825 & $\mathrm{E}$ & Italy & [46] \\
\hline Amabile Vecchioni & - & 1140 & - & 2950 & E & Italy & [46] \\
\hline Amoscatelado & $711-806$ & - & - & - & E & Spain & {$[60]$} \\
\hline Asgarabad & 710 & 652 & - & 3465 & $\mathrm{E}$ & Iran & {$[61]$} \\
\hline \multirow{2}{*}{ Aurora } & - & 1140 & - & 2750 & $\mathrm{E}$ & Italy & [46] \\
\hline & 1237 & 1296 & - & 2490-2812 & E & Italy & [62] \\
\hline Baracca & - & $1000-1140$ & - & 4680 & $\mathrm{E}$ & Italy & [46] \\
\hline Bebeco & - & $1030-1125$ & - & 3775 & $\mathrm{E}$ & Italy & [46] \\
\hline \multirow{4}{*}{ Bergeron } & - & 1225 & - & 4300 & $\mathrm{E}$ & Italy & [46] \\
\hline & 699 & 1176 & 64.8 & 4526 & E & Spain & [50] \\
\hline & 762 & 1134 & 61.7 & 5150 & E & Spain & [45] \\
\hline & - & $1122-1224$ & - & - & E & Serbia & [51] \\
\hline Blanc Rose & 1105-1185 & - & - & - & $\mathrm{E}$ & Spain & [60] \\
\hline \multirow{7}{*}{ Búlida } & 1050 & - & - & - & $\mathrm{E}$ & Spain & [63] \\
\hline & 950-983 & - & - & - & $\mathrm{E}$ & Spain & [60] \\
\hline & 976-1015 & - & - & - & $\mathrm{E}$ & Spain & [60] \\
\hline & $830-926$ & - & - & - & $\mathrm{E}$ & Spain & [60] \\
\hline & $1133 \pm 170$ & $1296 \pm 145$ & - & - & $\mathrm{E}$ & Spain & [64] \\
\hline & 560 & 968 & 53.8 & 5146 & E & Spain & [50] \\
\hline & 708 & 1048 & 56.4 & 5294 & $\mathrm{E}$ & Spain & [45] \\
\hline \multirow{2}{*}{ Cafona } & - & $1200 \pm 35$ & - & 3433 & E & Italy & [48] \\
\hline & - & $824-1515$ & - & $2499-5800$ & $\mathrm{E}$ & Spain & [48] \\
\hline \multirow{9}{*}{ Canino } & $\leq 750$ & - & - & - & E & Spain & [63] \\
\hline & $787-878$ & - & - & - & E & Spain & [60] \\
\hline & $<779$ & - & - & - & E & Spain & [60] \\
\hline & $771-779$ & - & - & - & E & Spain & [60] \\
\hline & - & 964-1370 & - & $2477-3087$ & $\mathrm{E}$ & Italy & [48] \\
\hline & - & $725-1350$ & - & $2547-6729$ & $\mathrm{E}$ & Spain & [48] \\
\hline & - & 1030 & - & 3275 & $\mathrm{E}$ & Italy & [46] \\
\hline & 532 & 806 & 45 & 5724 & $\mathrm{E}$ & Spain & [45] \\
\hline & 488 & 304 & 29.8 & - & E & $\begin{array}{l}\text { South } \\
\text { Africa }\end{array}$ & [45] \\
\hline
\end{tabular}


Table 2. Cont.

\begin{tabular}{|c|c|c|c|c|c|c|c|}
\hline \multirow{2}{*}{ Cultivar } & \multicolumn{3}{|c|}{ Chilling requirements } & \multirow{2}{*}{$\begin{array}{c}\text { Heat requirements } \\
\text { GDH }\end{array}$} & \multirow{2}{*}{ Method } & \multirow{2}{*}{ Loc. } & \multirow{2}{*}{ Ref } \\
\hline & $\mathrm{CH}$ & $\mathrm{CU}$ & $\mathrm{CP}$ & & & & \\
\hline Cegledy arany & - & $1122-1310$ & - & - & E & Serbia & [51] \\
\hline Charisma & 188 & 290 & 31.7 & - & E & $\begin{array}{l}\text { South } \\
\text { Africa }\end{array}$ & [45] \\
\hline Comice de Toulon & $806-878$ & - & - & - & E & Spain & {$[60]$} \\
\hline Corbato & $\leq 750$ & - & - & - & $\mathrm{E}$ & Spain & [63] \\
\hline \multirow{7}{*}{ Currot } & $\leq 750$ & - & - & - & $\mathrm{E}$ & Spain & [63] \\
\hline & $354-507$ & - & - & - & $\mathrm{E}$ & Spain & [60] \\
\hline & 267 & 596 & 34.3 & 5879 & E & Spain & [50] \\
\hline & - & 621 & 40.4 & $1611-2083$ & $\mathrm{E}$ & Italy & [49] \\
\hline & - & 634 & 38.8 & $2114-3168$ & E & Spain & [49] \\
\hline & - & $726-669$ & - & - & E & Italy & [65] \\
\hline & 409 & 642 & 37.8 & 5774 & E & Spain & [45] \\
\hline D'Alessandria & - & $1000-1140$ & - & 4150 & E & Italy & [46] \\
\hline Doctor Mascle & $592-711$ & - & - & - & $\mathrm{E}$ & Spain & [60] \\
\hline \multirow{2}{*}{ Dorada } & 594 & 1007 & 56.2 & 5079 & $\mathrm{E}$ & Spain & [50] \\
\hline & 720 & 1069 & 57.7 & 6189 & E & Spain & [45] \\
\hline Early Blush & 1407 & - & - & 2969 & $\mathrm{E}$ & Italy & [66] \\
\hline Galta Rocha & $372-592$ & - & - & - & $\mathrm{E}$ & Spain & [60] \\
\hline Giletano & $771-806$ & - & - & - & $\mathrm{E}$ & Spain & [60] \\
\hline \multirow{4}{*}{ Goldrich } & - & $950-1030$ & - & 3950 & E & Italy & [46] \\
\hline & - & 1084 & 65.2 & $485-913$ & E & Italy & [49] \\
\hline & & 992 & 58.6 & $2067-3431$ & $\mathrm{E}$ & Spain & [49] \\
\hline & - & $834-846$ & - & - & $\mathrm{E}$ & Serbia & [51] \\
\hline \multirow{2}{*}{ Harcot } & - & $1275-1530$ & - & $2267-2988$ & $\mathrm{E}$ & Italy & [48] \\
\hline & - & $920-1665$ & - & $3731-5355$ & E & Spain & [48] \\
\hline Hatif de Sig & $902-976$ & - & - & - & $\mathrm{E}$ & Spain & [60] \\
\hline Hoja de Parra & $668-787$ & - & - & - & $\mathrm{E}$ & Spain & [60] \\
\hline Koiska & 1050 & - & - & - & $\mathrm{E}$ & Spain & [63] \\
\hline \multirow{2}{*}{ Laycot } & 1214 & - & - & 3533 & $\mathrm{E}$ & Italy & [66] \\
\hline & 1045 & 1157 & - & $3252-3481$ & $\mathrm{E}$ & Italy & [62] \\
\hline \multirow{3}{*}{ Luicet } & 1150 & - & - & - & $\mathrm{E}$ & Spain & [63] \\
\hline & $1074-1140$ & - & - & - & $\mathrm{E}$ & Spain & [60] \\
\hline & $1058-1116$ & - & - & - & E & Spain & [60] \\
\hline Magyar kajski & - & $1122-1310$ & - & - & $\mathrm{E}$ & Serbia & [51] \\
\hline \multirow{4}{*}{ Moniqui } & 850 & - & - & - & E & Spain & [63] \\
\hline & $779-926$ & - & - & - & $E$ & Spain & [60] \\
\hline & $954 \pm 103$ & $1139 \pm 96$ & - & - & $\mathrm{E}$ & Spain & [64] \\
\hline & - & $930-1140$ & - & 3250 & $\mathrm{E}$ & Italy & [46] \\
\hline Moongold & - & 910 & - & 2712 & $\mathrm{E}$ & USA & [67] \\
\hline Moonpark & $1074-1105$ & - & - & - & E & Spain & [60] \\
\hline
\end{tabular}


Table 2. Cont.

\begin{tabular}{|c|c|c|c|c|c|c|c|}
\hline \multirow{2}{*}{ Cultivar } & \multicolumn{3}{|c|}{ Chilling requirements } & \multirow{2}{*}{$\begin{array}{c}\text { Heat requirements } \\
\text { GDH }\end{array}$} & \multirow{2}{*}{ Method } & \multirow{2}{*}{ Loc. } & \multirow{2}{*}{ Ref. } \\
\hline & $\mathrm{CH}$ & $\mathrm{CU}$ & $\mathrm{CP}$ & & & & \\
\hline \multirow{2}{*}{ Murciana } & 585 & 1009 & 55.9 & 4440 & $\mathrm{E}$ & Spain & {$[50]$} \\
\hline & 690 & 1030 & 55.6 & 5392 & E & Spain & {$[45]$} \\
\hline Ninfa & - & $834-846$ & - & - & $\mathrm{E}$ & Serbia & [51] \\
\hline \multirow{8}{*}{ Orangered } & 1587 & - & - & 3448 & E & Italy & [66] \\
\hline & - & $1450-1600$ & - & 2700 & E & Italy & {$[46]$} \\
\hline & 1481 & 1467 & - & $2654-3136$ & $\mathrm{E}$ & Italy & [62] \\
\hline & 738 & 1266 & 69.1 & 4362 & E & Spain & {$[50]$} \\
\hline & - & 902 & 55.5 & $2421-3398$ & E & Italy & [49] \\
\hline & - & 1146 & 67.2 & $1443-1505$ & $\mathrm{E}$ & Spain & [49] \\
\hline & 777 & 1172 & 64.3 & 4916 & $\mathrm{E}$ & Spain & {$[45]$} \\
\hline & 568 & 957 & 55.4 & - & E & $\begin{array}{l}\text { South } \\
\text { Africa }\end{array}$ & [45] \\
\hline \multirow{2}{*}{ Palsteyn } & 171 & 274 & 31.6 & - & $\mathrm{E}$ & $\begin{array}{l}\text { South } \\
\text { Africa }\end{array}$ & [45] \\
\hline & 413 & 631 & 37.1 & 6247 & $\mathrm{E}$ & Spain & [45] \\
\hline $\begin{array}{c}\text { Patriarca de Hueso } \\
\text { Dulce }\end{array}$ & $664-729$ & - & - & - & $\mathrm{E}$ & Spain & [60] \\
\hline \multirow{3}{*}{ Paviot } & 1050 & - & - & - & $\mathrm{E}$ & Spain & [63] \\
\hline & 995-1075 & - & - & - & $\mathrm{E}$ & Spain & [60] \\
\hline & $1148 \pm 148$ & $1318 \pm 145$ & - & - & $\mathrm{E}$ & Spain & [64] \\
\hline Perfection & - & 844 & - & 2593 & $\mathrm{E}$ & USA & [67] \\
\hline Perla & $1074-1105$ & - & - & - & $\mathrm{E}$ & Spain & [60] \\
\hline Phelps & - & 857 & - & 2206 & $\mathrm{E}$ & USA & [67] \\
\hline Pike & - & 895 & - & 2753 & $\mathrm{E}$ & USA & [67] \\
\hline Pisana & - & $1113-1122$ & - & - & $E$ & Serbia & [51] \\
\hline \multirow{4}{*}{ Polonais } & $1058-1116$ & - & - & - & E & Spain & [60] \\
\hline & - & $1175-1450$ & - & $2611-2823$ & $\mathrm{E}$ & Italy & [48] \\
\hline & - & $920-1665$ & - & $4047-5753$ & E & Spain & [48] \\
\hline & - & 1300 & - & 2850 & $\mathrm{E}$ & Italy & [46] \\
\hline \multirow{4}{*}{ Precoz de Colomer } & 950 & - & - & - & $\mathrm{E}$ & Spain & [63] \\
\hline & $<779$ & - & - & - & $\mathrm{E}$ & Spain & {$[60]$} \\
\hline & - & $1175-1250$ & - & $2503-2563$ & $\mathrm{E}$ & Italy & {$[48]$} \\
\hline & - & 690-1190 & - & $2320-5304$ & $\mathrm{E}$ & Spain & [48] \\
\hline Priana & - & $926 \pm 26$ & - & 2189 & E & Spain & [48] \\
\hline Rapareddu & - & 1250 & - & 2850 & $\mathrm{E}$ & Italy & [46] \\
\hline Re Umberto & - & $1126-1442$ & - & - & E & Serbia & [51] \\
\hline \multirow{2}{*}{ Rojo Pasión } & 531 & 917 & 51.2 & 4670 & E & Spain & [50] \\
\hline & 566 & 874 & 48.2 & 6078 & E & Spain & {$[45]$} \\
\hline Rouge de Rousillon & $950-1005$ & - & - & - & E & Spain & [60] \\
\hline \multirow{3}{*}{ Royal } & 875 & - & - & - & E & USA & [23] \\
\hline & 850 & - & - & - & $\mathrm{E}$ & Spain & [63] \\
\hline & $779-950$ & - & - & - & $\mathrm{E}$ & Spain & [60] \\
\hline
\end{tabular}


Table 2. Cont.

\begin{tabular}{|c|c|c|c|c|c|c|c|}
\hline \multirow{2}{*}{ Cultivar } & \multicolumn{3}{|c|}{ Chilling requirements } & \multirow{2}{*}{$\begin{array}{l}\text { Heat requirements } \\
\text { GDH }\end{array}$} & \multirow{2}{*}{ Method } & \multirow{2}{*}{ Loc. } & \multirow{2}{*}{ Ref } \\
\hline & $\mathrm{CH}$ & $\mathrm{CU}$ & $\mathrm{CP}$ & & & & \\
\hline \multirow{7}{*}{ San Castrese } & - & $964-1100$ & - & $1410-3289$ & E & Italy & [48] \\
\hline & - & $725 \pm 23$ & - & 4134 & E & Spain & [48] \\
\hline & 1044 & - & - & 3558 & E & Italy & {$[66]$} \\
\hline & - & $870-930$ & - & 3425 & E & Italy & [46] \\
\hline & 788 & 894 & - & $2705-3874$ & E & Italy & [62] \\
\hline & - & 880 & 54 & $1615-3326$ & E & Italy & [49] \\
\hline & - & 981 & 56.5 & $2116-2967$ & E & Spain & [49] \\
\hline S. Nicola Grosso & - & 1140 & - & 3350 & E & Italy & [46] \\
\hline Sarritzu I ${ }^{\circ}$ & - & $950-1140$ & - & 3950 & E & Italy & [46] \\
\hline \multirow{2}{*}{ Selene } & 590 & 1018 & 57.4 & 4078 & $\mathrm{E}$ & Spain & [50] \\
\hline & 705 & 1057 & 56.9 & 4605 & $\mathrm{E}$ & Spain & [45] \\
\hline Shakarpare & 862 & 746 & - & 3171 & $\mathrm{E}$ & Iran & [61] \\
\hline Shamlo & 1130 & 826 & - & 2987 & $\mathrm{E}$ & Iran & [61] \\
\hline Stark Early Orange & - & 1411 & 78.9 & & $\mathrm{E}$ & Italy & [49] \\
\hline Sundrop & - & $964-967$ & - & - & E & Serbia & [51] \\
\hline Sylred & - & $967-1019$ & - & - & E & Serbia & [51] \\
\hline Tabarze ghermez & 1130 & 826 & - & 2987 & $\mathrm{E}$ & Iran & [61] \\
\hline Tilton & 1000 & - & - & - & E & USA & [23] \\
\hline Tirynthos & & $935-1000$ & - & $1898-3289$ & $\mathrm{E}$ & Italy & [48] \\
\hline Tom Cot & - & $834-846$ & - & - & $\mathrm{E}$ & Serbia & [51] \\
\hline \multirow{2}{*}{ Tonda di Costiglione } & 1812 & - & - & 3643 & $\mathrm{E}$ & Italy & {$[66]$} \\
\hline & 1586 & 1561 & - & $3402-3696$ & $\mathrm{E}$ & Italy & [62] \\
\hline
\end{tabular}

Table 3. Chilling and heat requirements of Japanese apricot cultivars.

\begin{tabular}{cccccccc}
\hline \multirow{2}{*}{ Cultivar } & \multicolumn{2}{c}{ Chilling requirements } & Heat req. & Method & Loc. & Ref. \\
\hline $\mathbf{6 7}$ & - & - & 69 & 994 & E & China & {$[53]$} \\
\hline Baijiahe & - & - & 59 & 1316 & E & China & {$[53]$} \\
\hline Changnong17 & - & - & 69 & 994 & E & China & {$[53]$} \\
\hline Dabaimei & - & - & 56 & 1669 & E & China & {$[53]$} \\
\hline Dali & - & - & 40 & 1192 & E & China & {$[53]$} \\
\hline Danfenghou & - & - & 73 & 2054 & E & China & {$[53]$} \\
\hline Daqiandi & - & - & 66.5 & 1096 & E & China & {$[53]$} \\
\hline Daroumei & - & - & 38.5 & 1100 & E & China & {$[53]$} \\
\hline Dayezhugan & - & - & 73 & 1651 & E & China & {$[53]$} \\
\hline Dayu & - & - & 69 & 822 & E & China & {$[53]$} \\
\hline Dongqing & $875 \pm 147$ & $1054 \pm 256$ & $58 \pm 9$ & $1018 \pm 174$ & E & China & {$[52]$} \\
\hline Dongshanlimei & - & - & 50 & 1250 & E & China & {$[53]$} \\
\hline Ellching & 300 & - & - & - & E & Japan & {$[54]$} \\
\hline Fenghou & $1148 \pm 162$ & $1323 \pm 247$ & $73 \pm 8$ & $1697 \pm 1697$ & E & China & {$[52]$} \\
\hline Fenghualimei & - & - & 56 & 1533 & E & China & {$[53]$} \\
\hline
\end{tabular}


Table 3. Cont.

\begin{tabular}{|c|c|c|c|c|c|c|c|}
\hline \multirow{2}{*}{ Cultivar } & \multicolumn{3}{|c|}{ Chilling requirements } & \multirow{2}{*}{$\begin{array}{l}\text { Heat req. } \\
\text { GDH }\end{array}$} & \multirow{2}{*}{ Method } & \multirow{2}{*}{ Loc. } & \multirow{2}{*}{ Ref. } \\
\hline & $\mathrm{CH}$ & $\mathrm{CU}$ & $\mathrm{CP}$ & & & & \\
\hline Gaotianfenghou & - & - & 73 & 2054 & $\mathrm{E}$ & China & [53] \\
\hline Gaotiangmei & - & - & 64.5 & 1099 & $\mathrm{E}$ & China & [53] \\
\hline Guangdonghuangpi & - & - & 40 & 1192 & $\mathrm{E}$ & China & [53] \\
\hline Gucheng & - & - & 59 & 1383 & $\mathrm{E}$ & China & [53] \\
\hline Hangzhoubaimei & - & - & 60 & 1231 & $\mathrm{E}$ & China & [53] \\
\hline Henghe & - & - & 34 & 1287 & $\mathrm{E}$ & China & [53] \\
\hline Hongding & - & - & 69 & 860 & $\mathrm{E}$ & China & [53] \\
\hline Hongmei & - & - & 73 & 1835 & E & China & [53] \\
\hline Hongnong & - & - & 75 & 1675 & $\mathrm{E}$ & China & [53] \\
\hline Huangxiaoda & - & - & 56 & 1533 & $\mathrm{E}$ & China & [53] \\
\hline Huaxiangshi & - & - & 33.5 & 1072 & $\mathrm{E}$ & China & [53] \\
\hline Jiazhouxiaomei & - & - & 69 & 1733 & E & China & [53] \\
\hline Jiazhouzuixiao & - & - & 62 & 1116 & E & China & [53] \\
\hline Jietianmei & - & - & 77 & 2378 & E & China & [53] \\
\hline Jiuzhongmei & - & - & 66.5 & 1096 & $\mathrm{E}$ & China & [53] \\
\hline Lizimei & - & - & 69 & 977 & $\mathrm{E}$ & China & [53] \\
\hline Longyan & - & - & 73 & 1835 & $\mathrm{E}$ & China & [53] \\
\hline Lve & - & - & 29 & 1268 & $\mathrm{E}$ & China & [53] \\
\hline Nanhong & - & - & 50 & 1583 & $\mathrm{E}$ & China & [53] \\
\hline Nanko & 500 & - & - & - & E & Japan & [54] \\
\hline Pinzhimei & - & - & 73 & 1835 & $\mathrm{E}$ & China & [53] \\
\hline Qijiangxingmei & - & - & 56 & 1533 & $\mathrm{E}$ & China & [53] \\
\hline Qingjia2 & - & - & 75 & 1675 & $\mathrm{E}$ & China & [53] \\
\hline Qixingmei & - & - & 60 & 1232 & E & China & [53] \\
\hline Ruantiaohongmei & - & - & 73 & 1835 & $\mathrm{E}$ & China & [53] \\
\hline Shuangshuidaroumei & $239 \pm 84$ & $479 \pm 180$ & $26 \pm 7$ & $1235 \pm 77$ & $\mathrm{E}$ & China & {$[52]$} \\
\hline Shuangtaomei & - & - & 36.5 & 1079 & E & China & [53] \\
\hline Sichuangbaimei & - & - & 60 & 1231 & E & China & [53] \\
\hline Sichuanghuangmei & - & - & 60 & 1231 & $\mathrm{E}$ & China & [53] \\
\hline Sichuangqingmei & - & - & 78.5 & 2378 & $\mathrm{E}$ & China & [53] \\
\hline Siyuemei & - & - & 60 & 1231 & $\mathrm{E}$ & China & [53] \\
\hline Taihu1 & - & - & 69 & 977 & $\mathrm{E}$ & China & [53] \\
\hline Taihu3 & - & - & 50 & 1575 & E & China & [53] \\
\hline Taoxingmei & $332 \pm 110$ & $567 \pm 198$ & $\begin{array}{c}32 \pm \\
8\end{array}$ & $110 \pm 199$ & $\mathrm{E}$ & China & {$[52]$} \\
\hline Tengwulang & - & - & 78.5 & 2378 & $\mathrm{E}$ & China & [53] \\
\hline Tonglv & - & - & 73 & 1835 & $\mathrm{E}$ & China & [53] \\
\hline Touguhong & - & - & 62 & 986 & $\mathrm{E}$ & China & [53] \\
\hline Wanhong & - & - & 73 & 1835 & $\mathrm{E}$ & China & [53] \\
\hline Weishanzhong & - & - & 50 & 1574 & E & China & [53] \\
\hline Xianmimei & - & - & 40 & 1205 & E & China & [53] \\
\hline Xiaomei & - & - & 50 & 1369 & E & China & [53] \\
\hline Xiaoougongfen & - & - & 42.5 & 1037 & E & China & {$[53]$} \\
\hline
\end{tabular}


Table 3. Cont.

\begin{tabular}{|c|c|c|c|c|c|c|c|}
\hline \multirow{2}{*}{ Cultivar } & \multicolumn{3}{|c|}{ Chilling requirements } & \multirow{2}{*}{$\begin{array}{l}\text { Heat req. } \\
\text { GDH }\end{array}$} & \multirow{2}{*}{ Method } & \multirow{2}{*}{ Loc. } & \multirow{2}{*}{ Ref. } \\
\hline & $\mathrm{CH}$ & CU & $\mathrm{CP}$ & & & & \\
\hline Xiaoqing & - & - & 73 & 1432 & E & China & [53] \\
\hline Xiaoyezhugan & - & - & 56 & 1533 & E & China & {$[53]$} \\
\hline Xingnongxiaomei & - & - & 62 & 1116 & E & China & {$[53]$} \\
\hline Xiyeqing & $828 \pm 139$ & $1040 \pm 231$ & $\begin{array}{c}64 \pm \\
9\end{array}$ & $1179 \pm 230$ & E & China & [52] \\
\hline Xuemei & - & - & 69 & 1405 & $\mathrm{E}$ & China & [53] \\
\hline Yanglao1 & - & - & 59 & 1530 & E & China & [53] \\
\hline Yanglao2 & - & - & 34 & 1297 & E & China & [53] \\
\hline Yanglao3 & - & - & 56 & 1669 & E & China & [53] \\
\hline Yanhua & $1141 \pm 253$ & $1321 \pm 328$ & $\begin{array}{c}76 \pm \\
6\end{array}$ & $1250 \pm 213$ & E & China & [52] \\
\hline Yanzhimei & - & - & 69 & 977 & E & China & [53] \\
\hline Yeliqing & - & - & 69 & 994 & E & China & [53] \\
\hline Yingsu & - & - & 59 & 1463 & E & China & [53] \\
\hline Yinnafenghou & - & - & 73 & 2054 & $\mathrm{E}$ & China & {$[53]$} \\
\hline Yueshijie & - & - & 62 & 1116 & E & China & [53] \\
\hline Yunnanxingmei & - & - & 73 & 1835 & $\mathrm{E}$ & China & [53] \\
\hline Yuying & - & - & 62 & 1116 & E & China & [53] \\
\hline Zaohong & - & - & 50 & 1583 & E & China & [53] \\
\hline Zaohua & - & - & 56 & 1403 & $\mathrm{E}$ & China & [53] \\
\hline Zhizhimei & - & - & 69 & 1069 & $\mathrm{E}$ & China & [53] \\
\hline Zhonghong & - & - & 53 & 1542 & $\mathrm{E}$ & China & [53] \\
\hline
\end{tabular}

\subsection{Peach (P. persica)}

Peach is the stone fruit crop with higher economic importance. It has been confined traditionally to latitudes between $30^{\circ}$ and $50^{\circ}$ North and South [68], but in the last years, there is an increasing interest to expand it to warmer areas, including tropical and subtropical regions [68-70]. In recent decades, intense breeding has led to the release of an enormous number of cultivars of different types of fruit, including pubescent (peaches) or glabrous skin (nectarines), round or flat shape, white or yellow flesh, and freestone o clingstone [70] (Table 4). Several peach cultivars have been used to develop models in dormancy studies, both in experimental approaches to determine the date of breaking of endodormancy $[23,38,71]$ and in models to quantify chilling and forcing temperatures $[13,28,29,72]$. The DORMANCY-ASSOCIATED MAD-BOX (DAM) genes that regulate dormancy were first reported in an 'evergreen' peach mutant $[73,74]$.

This work compiles the chilling requirements of 216 cultivars, including seven flat peach cultivars, 25 nectarine cultivars and 172 peach cultivars, showing high differences in the range 239-536 $\mathrm{CH}$, 354-861 CU and 22.3-48.5 CP for flat peaches, 90-426 CH/ 45-1050CU/9-47CP for nectarines and 71-1390 $\mathrm{CH} / 5-1220 \mathrm{CU} / 1-1221.8 \mathrm{CP}$ for peaches. The heat requirements of 44 cultivars have been compiled, ranging from 5853 to $9338 \mathrm{GDH}$ for nectarine and between 3476 and $16493 \mathrm{GDH}$ for peach. 
Table 4. Chilling and heat requirements of peach cultivars.

\begin{tabular}{|c|c|c|c|c|c|c|c|c|}
\hline & \multirow[t]{2}{*}{ Cultivar } & \multicolumn{3}{|c|}{$\begin{array}{c}\text { Chilling } \\
\text { requirements }\end{array}$} & \multirow{2}{*}{$\begin{array}{c}\text { Heat } \\
\text { requirements } \\
\text { GDH }\end{array}$} & \multirow[t]{2}{*}{ Method } & \multirow[t]{2}{*}{ Loc. } & \multirow[t]{2}{*}{ Ref. } \\
\hline & & $\mathrm{CH}$ & CU & $\mathrm{CP}$ & & & & \\
\hline \multirow{7}{*}{ Flat peach } & Carioca & 368 & 582 & 35.5 & - & $E$ & Spain & [75] \\
\hline & Siroco 10 & 305 & 480 & 28.9 & - & $E$ & Spain & [75] \\
\hline & Siroco 5 & 239 & 355 & 22.3 & - & E & Spain & [75] \\
\hline & Sweet Cap & 536 & 86 & 47.6 & - & $\mathrm{E}$ & Spain & [75] \\
\hline & UFO 2 & 432 & 681 & 40.2 & - & E & Spain & [75] \\
\hline & UFO 3 & 451 & 741 & 43.8 & - & E & Spain & [75] \\
\hline & UFO 4 & 484 & 803 & 48.5 & - & E & Spain & [75] \\
\hline \multirow{26}{*}{ Nectarine } & Caldessi 2000 & 316 & 210 & 33 & 9002 & S & Argentina & [76] \\
\hline & Carolina & 326 & - & - & - & S & Argentina & [77] \\
\hline & Cheonhong & - & 800 & - & - & $S$ & Korea & [78] \\
\hline & Collins & - & 950 & - & - & S & Korea & [78] \\
\hline & Cortez & - & 750 & - & - & $\mathrm{S}$ & Korea & [78] \\
\hline & Derby & - & 750 & - & - & $\mathrm{S}$ & Korea & {$[78]$} \\
\hline & Earliscarlet & - & 800 & - & - & $S$ & Korea & [78] \\
\hline & Early Giant & 342 & 249 & 46 & 8677 & $\mathrm{~S}$ & Argentina & [76] \\
\hline & Fantasia & - & 750 & - & - & $\mathrm{S}$ & Korea & {$[78]$} \\
\hline & Firebrite & 308 & 198 & 34 & 7498 & $\mathrm{~S}$ & Argentina & [76] \\
\hline & Flavortop & - & 750 & - & - & $S$ & Korea & {$[78]$} \\
\hline & Garden State & - & 1050 & - & - & S & Korea & [78] \\
\hline & Hahong & - & 700 & - & - & S & Korea & [78] \\
\hline & Hardired & - & 950 & - & - & S & Korea & {$[78]$} \\
\hline & \multirow{2}{*}{ Lara } & 350 & - & - & - & $\mathrm{S}$ & Argentina & [77] \\
\hline & & 93 & 47 & 9 & 9338 & $\mathrm{~S}$ & Argentina & [76] \\
\hline & María Anna & 426 & 392 & 46 & 5853 & $\mathrm{~S}$ & Argentina & [76] \\
\hline & María Lucía & 413 & 244 & 47 & 6777 & $\mathrm{~S}$ & Argentina & [76] \\
\hline & May Glo & 98 & 84 & 12 & 8242 & S & Argentina & [76] \\
\hline & May Grand & - & 800 & - & - & S & Korea & [78] \\
\hline & Redgold & - & 850 & - & - & S & Korea & [78] \\
\hline & Roseprincess & 313 & 207 & 33 & 9000 & S & Argentina & [76] \\
\hline & Suhong & - & 700 & - & - & $\mathrm{S}$ & Korea & {$[78]$} \\
\hline & Sunfre & - & 500 & - & - & S & Korea & {$[78]$} \\
\hline & Sungem & - & 425 & - & - & S & Korea & {$[78]$} \\
\hline & Sunraycer & 90 & 45 & 10 & 9086 & S & Argentina & {$[76]$} \\
\hline \multirow{7}{*}{ Peach } & Afterglow & 750 & - & - & - & S & USA & {$[28]$} \\
\hline & Akatsuki & 1176 & 1074 & - & 5675 & E & Japan & [79] \\
\hline & Anjiry Asali & 862 & 746 & - & 4232 & E & Iran & [61] \\
\hline & Anjiry Zafarany & 973 & 805 & - & 4099 & E & Iran & [61] \\
\hline & Armking & - & 600 & - & - & $\mathrm{S}$ & Korea & [78] \\
\hline & Autumnglo & - & 950 & - & - & S & Korea & [78] \\
\hline & Babygold 5 & 498 & 364 & 53 & 8505 & S & Argentina & [76] \\
\hline
\end{tabular}


Table 4. Cont.

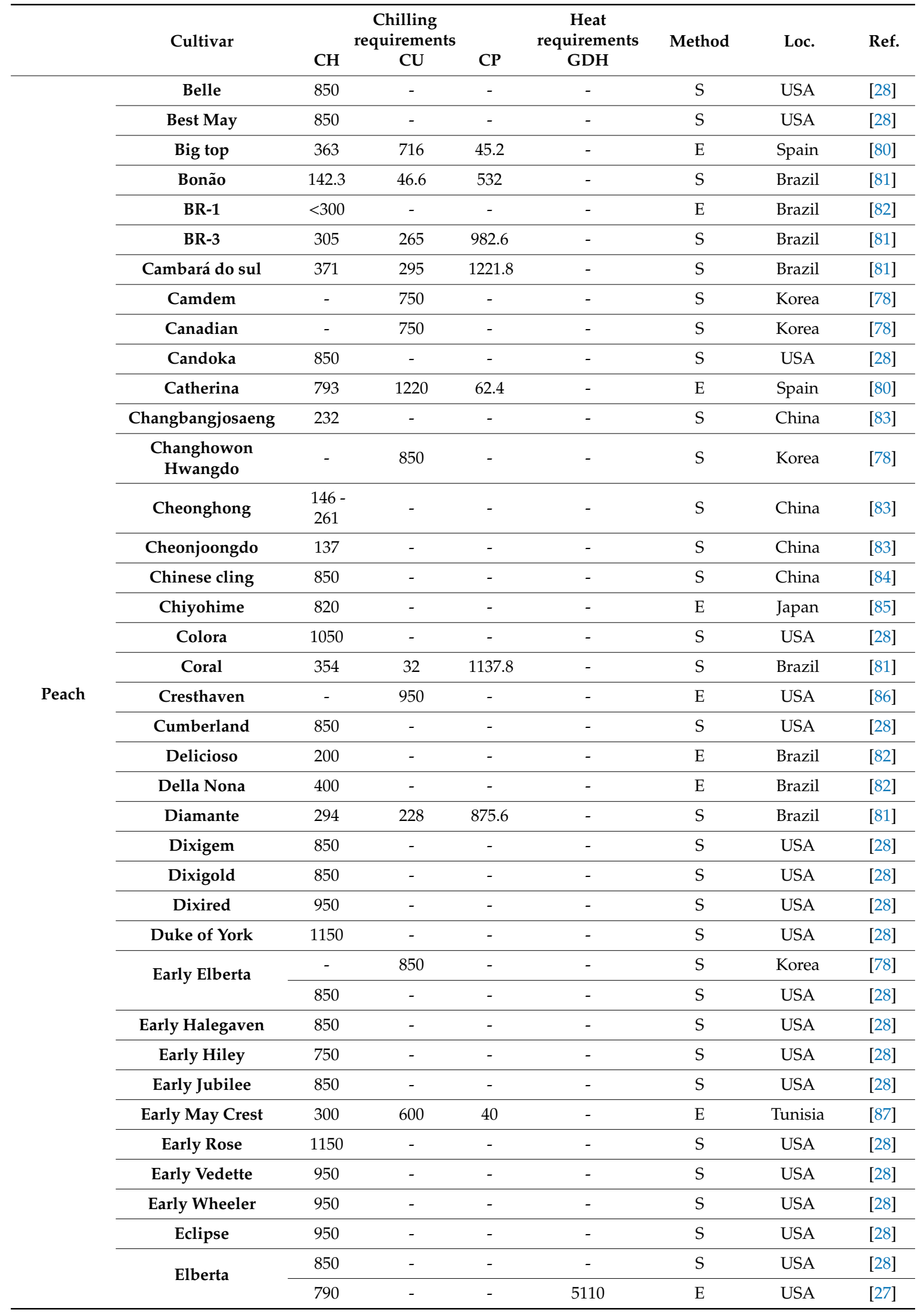


Table 4. Cont.

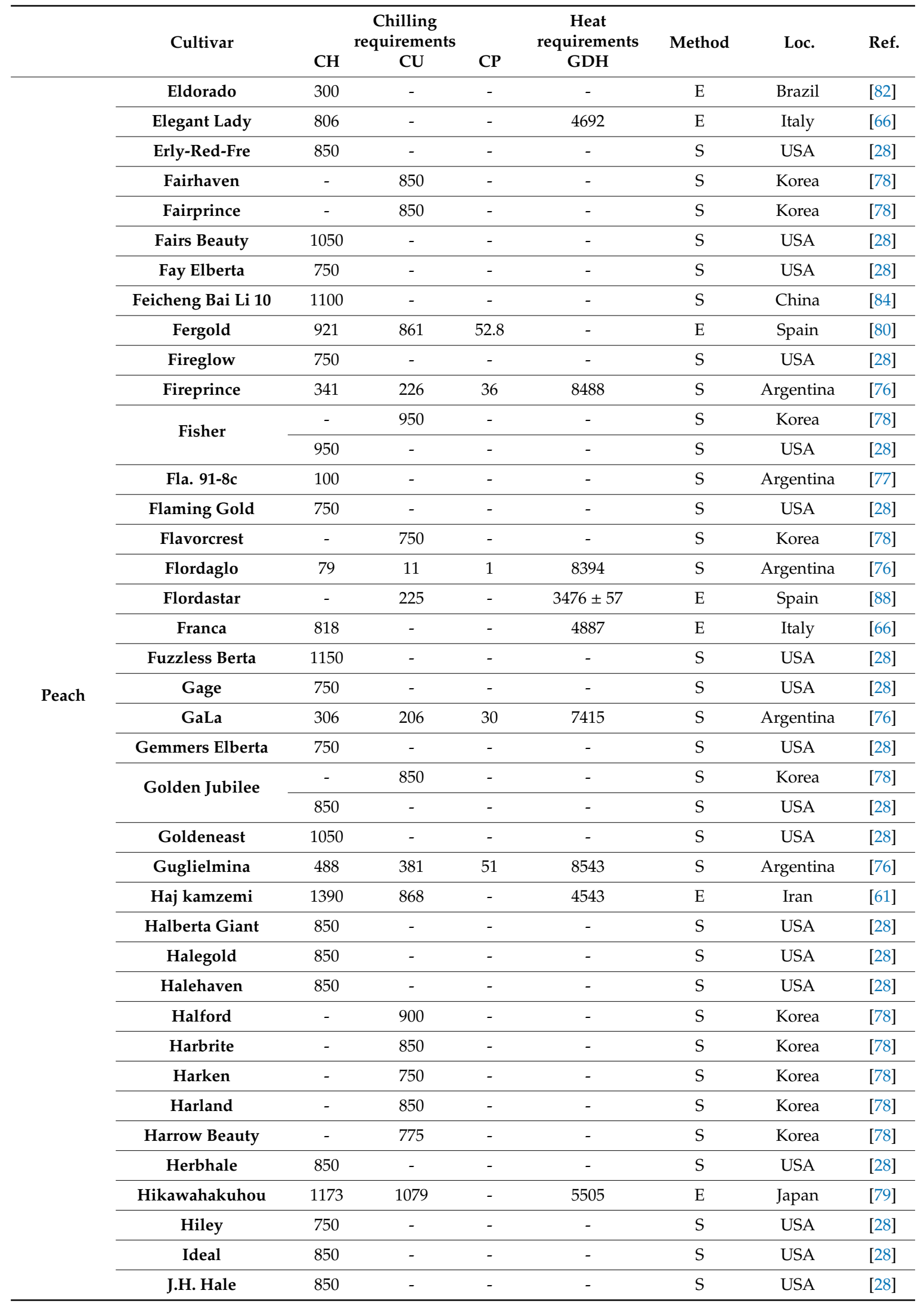


Table 4. Cont.

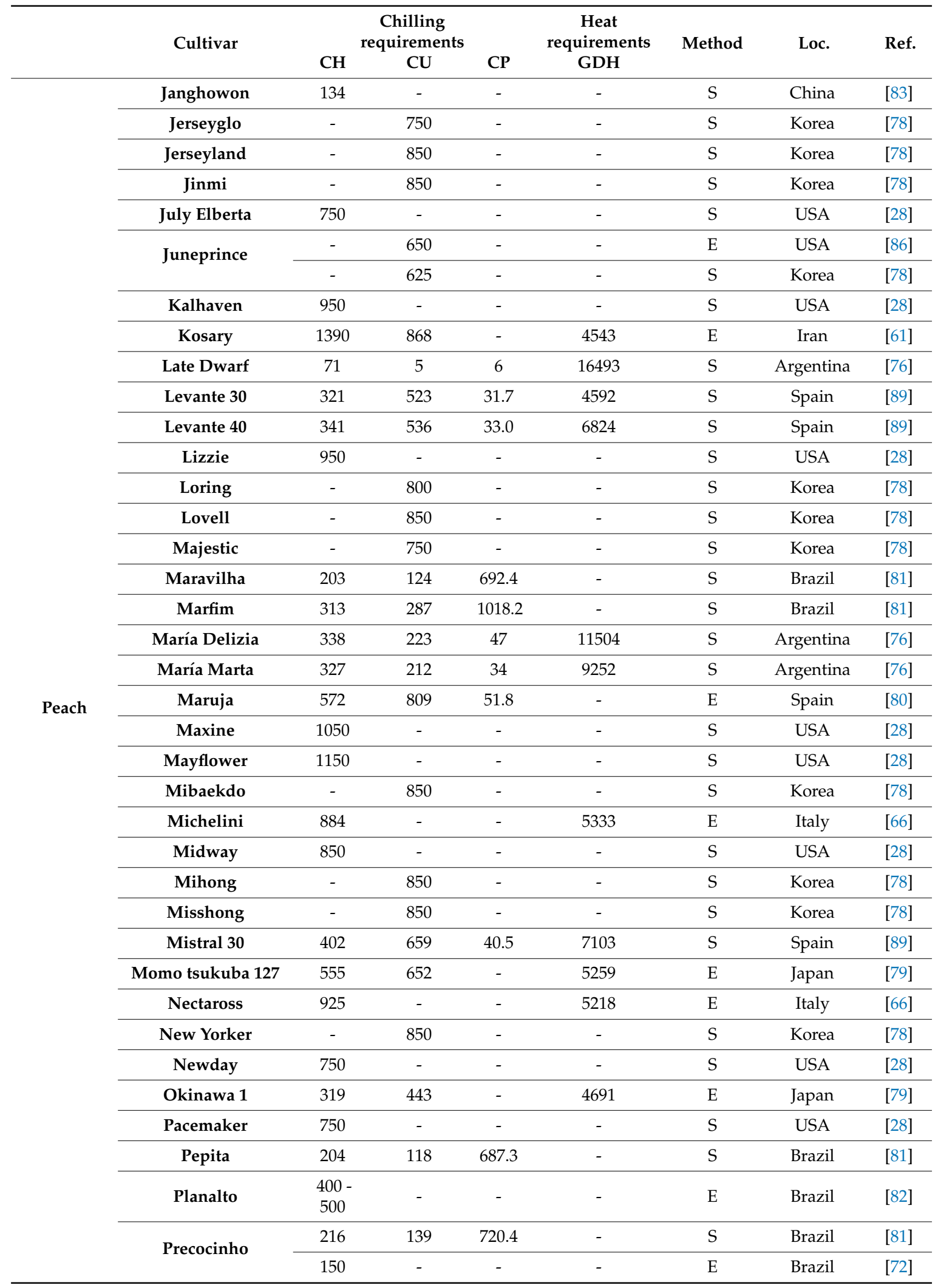


Table 4. Cont.

\begin{tabular}{|c|c|c|c|c|c|c|c|c|}
\hline & \multirow[t]{2}{*}{ Cultivar } & \multicolumn{3}{|c|}{$\begin{array}{c}\text { Chilling } \\
\text { requirements }\end{array}$} & \multirow{2}{*}{$\begin{array}{c}\text { Heat } \\
\text { requirements } \\
\text { GDH }\end{array}$} & \multirow[t]{2}{*}{ Method } & \multirow[t]{2}{*}{ Loc. } & \multirow[t]{2}{*}{ Ref. } \\
\hline & & $\mathrm{CH}$ & $\mathrm{CU}$ & $\mathrm{CP}$ & & & & \\
\hline & $\begin{array}{c}\text { Qingzhou Bai Pi } \\
\text { Mi Tao }\end{array}$ & 1100 & - & - & - & $S$ & China & [84] \\
\hline & \multirow{2}{*}{ Raritan Rose } & - & 1050 & - & - & $\mathrm{S}$ & Korea & [78] \\
\hline & & 950 & - & - & - & $\mathrm{S}$ & USA & {$[28]$} \\
\hline & \multirow{2}{*}{ Red Globed } & - & 850 & - & - & E & USA & [86] \\
\hline & & - & 850 & - & - & $S$ & Korea & {$[78]$} \\
\hline & Redelberta & 750 & - & - & - & $S$ & USA & {$[28]$} \\
\hline & \multirow{2}{*}{ Redhaven } & 850 & - & - & - & $S$ & USA & {$[28]$} \\
\hline & & 870 & - & - & 4922 & $E$ & USA & [27] \\
\hline & Redheaven & - & 950 & - & - & $\mathrm{S}$ & Korea & {$[78]$} \\
\hline & Redrose & 850 & - & - & - & $S$ & USA & [28] \\
\hline & Reliance & - & 1050 & - & - & $S$ & Korea & [78] \\
\hline & Rich Lady & 73 & 6 & 11 & 14086 & $\mathrm{~S}$ & Argentina & {$[76]$} \\
\hline & Richhaven & - & 950 & - & - & $\mathrm{S}$ & Korea & [78] \\
\hline & Rio Oso Gem & 850 & - & - & - & $S$ & USA & [28] \\
\hline & Riograndense & 300 & - & - & - & E & Brazil & [82] \\
\hline & Rosa del West & 434 & 378 & 51 & 9879 & $\mathrm{~S}$ & Argentina & {$[76]$} \\
\hline & Ruiguang 03 & - & 777 & - & - & $\mathrm{S}$ & Korea & {$[78]$} \\
\hline & Salberta & 850 & - & - & - & $S$ & USA & [28] \\
\hline & Salwy & 1050 & - & - & - & $S$ & USA & {$[28]$} \\
\hline & Sentry & - & 875 & - & - & $S$ & Korea & {$[78]$} \\
\hline \multirow[t]{19}{*}{ Peach } & Shipper Late Red & 850 & - & - & - & $S$ & USA & {$[28]$} \\
\hline & Siroco 5 & 246 & 427 & 25.8 & 7025 & $\mathrm{~S}$ & Spain & [89] \\
\hline & Siroco 20 & 344 & 561 & 35.8 & 7384 & $S$ & Spain & [89] \\
\hline & Siroco 30 & 308 & 539 & 31.2 & 6815 & $S$ & Spain & [89] \\
\hline & Siroco 40 & 310 & 509 & 30.3 & 7463 & $\mathrm{~S}$ & Spain & [89] \\
\hline & Siroco 43 & 370 & 593 & 36.4 & 6552 & S & Spain & [89] \\
\hline & Southland & 750 & - & - & - & $S$ & USA & [28] \\
\hline & Spring Belle & 650 & - & - & - & $S$ & Spain & [90] \\
\hline & Spring Lady & 331 & 625 & 40.8 & - & E & Spain & {$[80]$} \\
\hline & Springtime & - & 650 & - & - & $\mathrm{S}$ & Korea & {$[78]$} \\
\hline & Stark Red Gold & 898 & - & - & 5188 & $E$ & Italy & [66] \\
\hline & Starking Delicious & - & 750 & - & - & $S$ & Korea & {$[78]$} \\
\hline & Sullivan & 850 & - & - & - & $\mathrm{S}$ & USA & {$[28]$} \\
\hline & Summercrest & 950 & - & - & - & $\mathrm{S}$ & USA & {$[28]$} \\
\hline & Sunglo & - & 850 & - & - & $\mathrm{S}$ & Korea & {$[78]$} \\
\hline & Sunhigh & 750 & - & - & - & $S$ & USA & {$[28]$} \\
\hline & \multirow{2}{*}{ Sunland } & - & 750 & - & - & $\mathrm{E}$ & USA & [86] \\
\hline & & - & 750 & - & - & S & Korea & {$[78]$} \\
\hline & Triogem & 850 & - & - & - & $\mathrm{S}$ & USA & {$[28]$} \\
\hline
\end{tabular}


Table 4. Cont.

\begin{tabular}{|c|c|c|c|c|c|c|c|c|}
\hline & \multirow[t]{2}{*}{ Cultivar } & \multicolumn{3}{|c|}{$\begin{array}{c}\text { Chilling } \\
\text { requirements }\end{array}$} & \multirow{2}{*}{$\begin{array}{c}\text { Heat } \\
\text { requirements } \\
\text { GDH }\end{array}$} & \multirow[t]{2}{*}{ Method } & \multirow[t]{2}{*}{ Loc. } & \multirow[t]{2}{*}{ Ref. } \\
\hline & & $\mathrm{CH}$ & $\mathrm{CU}$ & $\mathrm{CP}$ & & & & \\
\hline \multirow{16}{*}{ Peach } & Tropic Beauty & - & 150 & - & - & $S$ & Korea & [78] \\
\hline & Tropic Snow & - & 200 & - & - & $S$ & Korea & [78] \\
\hline & Turmalina & 250 & 184 & 801.8 & - & $S$ & Brazil & [81] \\
\hline & Up-to-date & 850 & - & - & - & $S$ & USA & [28] \\
\hline & Valiant & 850 & - & - & - & $S$ & USA & [28] \\
\hline & Vedette & 1050 & - & - & - & $S$ & USA & [28] \\
\hline & Veeglo & - & 950 & - & - & $S$ & Korea & [78] \\
\hline & Veteran & 1050 & - & - & - & $S$ & USA & [28] \\
\hline & Vivid & - & 950 & - & - & $S$ & Korea & [78] \\
\hline & Worlds Earliest & 750 & - & - & - & $S$ & USA & [28] \\
\hline & Yanguang & - & 780 & - & - & $S$ & Korea & [78] \\
\hline & Youmyeong & $\begin{array}{l}150- \\
277\end{array}$ & - & - & - & S & China & [83] \\
\hline & Yu Hua Lu & 800 & - & - & - & $S$ & China & [84] \\
\hline & Yumi & - & 800 & - & - & $S$ & Korea & [78] \\
\hline & Yumyeong & - & 850 & - & - & $S$ & Korea & [78] \\
\hline & Zoud Ras & 1130 & 826 & - & 4384 & E & Iran & [61] \\
\hline
\end{tabular}

\subsection{European and Japanese plum (P. domestica and P. salicina).}

World production of plums increased by almost $20 \%$ in the last 10 years (from 9.5 million tons in 2007 to 12 million tons in 2017) [2]. These data include European plums, Japanese plums and hybrids between different Prunus sp. In spite of the economic importance of this crop, temperature requirements are little studied, with data available for only nine cultivars of European plum (Table 5) [91] and 16 cultivars of Japanese plum (Table 6) from two studies performed in Spain [31,91]. The experimental procedure used was slightly different between studies, with variation in the temperature of the growing chamber and in the growth evaluation procedure.

European plums are cultivated in colder climates [92], showing higher chilling requirements (579-1323 CH) than Japanese plums $(118-685 \mathrm{CH}$ ) (Tables 5 and 6). The chilling requirements of European plum ranged from 579-678 CH for 'Reine Claude d'Oullins' to $1116-1323 \mathrm{CH}$ for 'Reine Claude Noir' [93]. There is no data available on heat requirements in this species.

The Japanese plum cultivars with lower chilling requirements are 'Methley' (118-239 CH [91] and 'Pioneer' (181-231 CH / 297-358 CU / 18.4-27.6 CP), which also showed the lower values of heat requirements (5261-6720 GDH) [31]. 'Songold' showed higher values both for chilling (561-630 CH/ 974-1001 CU / 60.4-61.1 CP) and heat requirements (8588-10034 GDH). There are only data from different reports for 'Golden Japan' and 'Santa Rosa', showing high differences between them (Table 6). 
Table 5. Chilling and heat requirements of European plum cultivars.

\begin{tabular}{cccccccc}
\hline \multirow{2}{*}{ Cultivar } & \multicolumn{2}{c}{ Chilling requirements } & Heat req. & \multirow{2}{*}{ Method } & Loc. & \multirow{2}{*}{ Ref. } \\
\hline Beauty Plum of Catalogne & $\mathbf{6 7 8 - 8 1 9}$ & - & - & - & E & Spain & {$[91]$} \\
\hline Coe's Golden Drop & $984-1157$ & - & - & - & E & Spain & {$[91]$} \\
\hline Real de Calahorra & $976-1029$ & - & - & - & E & Spain & {$[91]$} \\
\hline Reine Claude de Bavay & $984-1157$ & - & - & - & E & Spain & {$[91]$} \\
\hline Reine Claudie Noir & $1116-1323$ & - & - & - & E & Spain & {$[91]$} \\
\hline Reine Claude d'Oullins & $579-678$ & - & - & - & E & Spain & {$[91]$} \\
\hline Reine Claude Verte & $976-1275$ & - & - & - & E & Spain & {$[91]$} \\
\hline Reine Claude Violetta d'Agen & $819-984$ & - & - & - & E & Spain & {$[91]$} \\
\hline Reine Claude Washington & $976-1275$ & - & - & - & E & Spain & {$[91]$} \\
\hline
\end{tabular}

Table 6. Chilling and heat requirements of Japanese plum cultivars.

\begin{tabular}{|c|c|c|c|c|c|c|c|}
\hline \multirow{2}{*}{ Cultivar } & \multicolumn{3}{|c|}{ Chilling requirements } & \multirow{2}{*}{$\begin{array}{l}\text { Heat req. } \\
\text { GDH }\end{array}$} & \multirow[b]{2}{*}{ Method } & \multirow[b]{2}{*}{ Loc. } & \multirow{2}{*}{ Ref. } \\
\hline & $\mathrm{CH}$ & $\mathrm{CU}$ & $\mathrm{CP}$ & & & & \\
\hline Angeleno & $434-447$ & $750-779$ & $39.7-50.1$ & $7300-8180$ & $\mathrm{E}$ & Spain & [31] \\
\hline Apex & $486-678$ & - & - & - & $\mathrm{E}$ & Spain & [91] \\
\hline Black Diamond & $389-432$ & $630-750$ & $38.7-47.1$ & $6994-8068$ & $\mathrm{E}$ & Spain & [31] \\
\hline Black Splendor & $213-324$ & $437-605$ & $29.1-31.2$ & $5744-6705$ & $\mathrm{E}$ & Spain & [31] \\
\hline Burbank & $486-678$ & - & - & - & $\mathrm{E}$ & Spain & [91] \\
\hline Formosa & $486-678$ & - & - & - & $\mathrm{E}$ & Spain & [91] \\
\hline Fortune & $436-447$ & $750-769$ & $39.7-46.7$ & $6681-8506$ & $\mathrm{E}$ & Spain & [31] \\
\hline Golden Globe & $473-685$ & $872-1053$ & $44.8-63.5$ & $7300-9151$ & $\mathrm{E}$ & Spain & [31] \\
\hline \multirow{2}{*}{ Golden Japan } & $118-287$ & - & - & - & E & Spain & [91] \\
\hline & $384-454$ & $701-829$ & $35.4-52.1$ & 6939-7855 & $\mathrm{E}$ & Spain & [31] \\
\hline Laetitia & $436-454$ & $750-829$ & $39.7-52.1$ & $7894-8020$ & $\mathrm{E}$ & Spain & [31] \\
\hline Methley & $118-239$ & - & - & - & $\mathrm{E}$ & Spain & [91] \\
\hline Pioneer & $181-231$ & $297-358$ & $18.4-27.6$ & $5261-6720$ & $\mathrm{E}$ & Spain & [31] \\
\hline Red Beauty & $265-369$ & $500-688$ & $25.6-46$ & $6727-7183$ & $\mathrm{E}$ & Spain & [31] \\
\hline \multirow{2}{*}{ Santa Rosa } & $372-627$ & - & - & - & E & Spain & [91] \\
\hline & $436-459$ & $750-829$ & $41.8-52.1$ & 6591-9099 & $\mathrm{E}$ & Spain & [31] \\
\hline Songold & $561-630$ & 974-1001 & $60.4-61.1$ & 8588-10034 & E & Spain & [31] \\
\hline Wickson & $345-627$ & - & - & - & E & Spain & [91] \\
\hline
\end{tabular}

\subsection{Sweet and Sour Cherry (P. avium and P. cerasus)}

Sweet cherries are mainly produced in Mediterranean countries as Turkey ( 0.63 million $\mathrm{t} / \mathrm{year}$ ), Italy ( 0.12 million $t$ /year), Spain ( 0.11 million $t /$ year) and Greece ( 0.90 million $t / y e a r)$, in Middle Eastern countries such as Iran (0.14 million $\mathrm{t} /$ year) and Uzbekistan ( 0.14 million $\mathrm{t} / \mathrm{year})$, in the United States (0.40 million $\mathrm{t} / \mathrm{year})$, and in Chile (0.13 million $\mathrm{t} /$ year) [2]. Chilling requirements are available for 53 cultivars [34,94-97], while the heat requirements were only calculated for 16 of them (Table 7) [34,96]. Chilling requirements are mainly quantified with $\mathrm{CH}$ in 48 cultivars [94,96] that range from $176 \mathrm{CH}$ of 'Cristobalina' [96] to more than $1100 \mathrm{CH}$ for 'Garrafal de Lérida', 'Hedelfinger', 'Lambert', 'Napoleón', and 'Vignola' [94]. Data available in CU in 21 cultivars range between $94 \mathrm{CU}$ for 'Lapins' and 'Larian' [97] and $1559 \pm 53 \mathrm{CU}$ for 'Skeena' [95], and 8 cultivars in CP from to 30.4 CP for 'Cristobalina' to 57.6 CP for 'Marvin' [96]. Chilling requirements for 'Burlat' and 'Cristobalina' were experimentally analyzed in two studies, resulting in higher values in the north of Spain ('Burlat' $900-100 \mathrm{CH}$ and 'Cristobalina' <800 CH) [94] than in the Mediterranean area ('Burlat' $618 \mathrm{CH}$ and 'Cristobalina' 
$176 \mathrm{CH}$ ) [96]. Estimations of heat requirements showed high variation between cultivars and locations: $3473 \pm 1236$ GDH for 'Schneider' in Germany [34], and 15500-16000 GDH are for 'Lapins', 'Larian', and '0900 Ziraat' in Turkey [97].

The experimental methodology [94-97] showed slight differences among studies: the frequency of field sampling (every 2 [97] or 7 [94] days, or every 50-100 CU [96]), the temperature of the chamber $\left(24 \pm 1^{\circ} \mathrm{C}[96,97]\right.$ or $20 \pm 1^{\circ} \mathrm{C}$ [94]), the period in the chamber (7 [94,95], 10 [96] or 21 [97] days), and also in the bud growth evaluation (dry weight [94], fresh weight [95], or phenology [96,97]). A statistical methodology was developed based on 'Schneiders' in Germany [34].

Sour cherry requirements have been poorly studied. There is only data of cultivar Montmorency, which showed $954 \mathrm{CU}$ and $6130 \mathrm{GDH}$ [42] (Table 7).

Table 7. Chilling and heat requirements of sour and sweet cherry cultivars.

\begin{tabular}{|c|c|c|c|c|c|c|c|}
\hline \multirow{2}{*}{ Cultivar } & \multicolumn{3}{|c|}{ Chilling requirements } & \multirow{2}{*}{$\begin{array}{c}\text { Heat } \\
\text { requirements } \\
\text { GDH }\end{array}$} & \multirow{2}{*}{ Method } & \multirow{2}{*}{ Loc. } & \multirow{2}{*}{ Ref. } \\
\hline & $\mathrm{CH}$ & CU & $\mathrm{CP}$ & & & & \\
\hline Ambrunés & $1000-1100$ & - & - & - & $\mathrm{E}$ & Spain & [94] \\
\hline \multirow{2}{*}{ Bing } & $1000-1100$ & - & - & - & $\mathrm{E}$ & Spain & [94] \\
\hline & - & $1082 \pm 27$ & - & - & $\mathrm{E}$ & Spain & [95] \\
\hline Brooks & 411.5 & 556 & 36.7 & 7863.2 & $\mathrm{E}$ & Spain & [96] \\
\hline \multirow{4}{*}{ Burlat } & $900-1000$ & - & - & - & $\mathrm{E}$ & Spain & [94] \\
\hline & 618 & 806 & 48 & 8750.2 & $\mathrm{E}$ & Spain & [96] \\
\hline & - & $981 \pm 83$ & - & - & $\mathrm{E}$ & Spain & [95] \\
\hline & - & - & 86 & - & $\mathrm{E}$ & Spain & [98] \\
\hline Cherovina & $900-1000$ & - & - & - & $\mathrm{E}$ & Spain & [94] \\
\hline \multirow{4}{*}{ Cristobalina } & $<800$ & - & - & - & $\mathrm{E}$ & Spain & [94] \\
\hline & 176 & 397 & 30.4 & 9195 & $\mathrm{E}$ & Spain & [96] \\
\hline & - & $687 \pm 83$ & - & - & $\mathrm{E}$ & Spain & [95] \\
\hline & - & - & 29 & - & E & Spain & [98] \\
\hline Daiber & $1000-1100$ & - & - & - & $\mathrm{E}$ & Spain & [94] \\
\hline Earlise & - & $981 \pm 83$ & - & - & $\mathrm{E}$ & Spain & [95] \\
\hline Early Rivers & $800-900$ & - & - & - & $\mathrm{E}$ & Spain & [94] \\
\hline Fertard & - & - & 101 & - & $\mathrm{E}$ & Spain & [98] \\
\hline Garrafal de Lérida & $>1100$ & - & - & - & $\mathrm{E}$ & Spain & [94] \\
\hline Guillaume & $1000-1100$ & - & - & - & $\mathrm{E}$ & Spain & [94] \\
\hline Hedelfingen & $>1100$ & - & - & - & $\mathrm{E}$ & Spain & [94] \\
\hline Jaboulay & $1000-1100$ & - & - & - & E & Spain & [94] \\
\hline Jarandilla & $>1100$ & - & - & - & $\mathrm{E}$ & Spain & [94] \\
\hline Kordia & $700-750$ & 150 & - & 14000 & $\mathrm{E}$ & Turkey & [97] \\
\hline Lambert & $>1100$ & - & - & - & E & Spain & [94] \\
\hline Lampé (Ramillete) & $900-1000$ & - & - & - & E & Spain & [94] \\
\hline Lapins & $400-450$ & 94 & & $15500-16000$ & E & Turkey & [97] \\
\hline Larian & 450 & 94 & & $15500-16000$ & E & Turkey & [97] \\
\hline Marmotte & $1000-1100$ & - & - & - & $\mathrm{E}$ & Spain & [94] \\
\hline Marvin & 788 & 1002 & 57.6 & 9450 & $\mathrm{E}$ & Spain & [96] \\
\hline Merton Glory & $900-1000$ & - & - & - & $\mathrm{E}$ & Spain & [94] \\
\hline Mollar de cáceres & $900-1000$ & - & - & - & $\mathrm{E}$ & Spain & [94] \\
\hline Moreau & $1000-1100$ & - & - & - & $\mathrm{E}$ & Spain & [94] \\
\hline Nafrina & $500-550$ & 120 & - & $15000-15500$ & $E$ & Turkey & [97] \\
\hline Napoleón & $>1100$ & - & - & - & $\mathrm{E}$ & Spain & [94] \\
\hline New Star & 709 & 909 & 53.5 & 8257 & $E$ & Spain & [96] \\
\hline
\end{tabular}


Table 7. Cont.

\begin{tabular}{|c|c|c|c|c|c|c|c|}
\hline \multirow{2}{*}{ Cultivar } & \multicolumn{3}{|c|}{ Chilling requirements } & \multirow{2}{*}{$\begin{array}{c}\text { Heat } \\
\text { requirements } \\
\text { GDH }\end{array}$} & \multirow{2}{*}{ Method } & \multirow[t]{2}{*}{ Loc. } & \multirow[t]{2}{*}{ Ref } \\
\hline & $\mathbf{C H}$ & $\mathrm{CU}$ & $\mathrm{CP}$ & & & & \\
\hline Noir de Guben & $600-650$ & 110 & - & $14000-14500$ & E & Turkey & [97] \\
\hline Pico Colorado & $1000-1100$ & - & - & - & $\mathrm{E}$ & Spain & [94] \\
\hline Producta & $1000-1100$ & - & - & - & $\mathrm{E}$ & Spain & [94] \\
\hline Ramón Oliva & 900-1000 & - & - & - & E & Spain & [94] \\
\hline Regina & - & - & 86 & - & E & Spain & [98] \\
\hline Reverchon & $1000-1100$ & - & - & - & E & Spain & [94] \\
\hline Ripolla & $800-900$ & - & - & - & E & Spain & [94] \\
\hline Ruby & 618 & 806 & 48 & 7326 & E & Spain & [96] \\
\hline Somerset & 618 & 806 & 48 & 8625.2 & E & Spain & [96] \\
\hline Schneiders & $698 \pm 151$ & $794 \pm 17$ & $\begin{array}{l}45.7 \\
\pm 5.4\end{array}$ & $3473 \pm 1236$ & S & Germany & {$[34]$} \\
\hline Skeena & - & $1559 \pm 63$ & - & - & E & Spain & [95] \\
\hline Stark Hardy Giant & $1000-1100$ & - & - & - & E & Spain & {$[94]$} \\
\hline Summit & 650 & 125 & - & 15000 & E & Turkey & [97] \\
\hline Sunburst & $650-700$ & 141 & - & 14000-14500 & E & Turkey & [97] \\
\hline Taleguera Brillante & $1000-1100$ & - & - & - & $\mathrm{E}$ & Spain & [94] \\
\hline Temprana de Sot & $1000-1100$ & - & - & - & E & Spain & [94] \\
\hline Tigré & $900-1000$ & - & - & - & E & Spain & [94] \\
\hline Van & $1000-1100$ & - & - & - & E & Spain & [94] \\
\hline Vernon & $1000-1100$ & - & - & - & $\mathrm{E}$ & Spain & [94] \\
\hline Vignola & $>1100$ & - & - & - & $\mathrm{E}$ & Spain & [94] \\
\hline Villareta & 900-1000 & - & - & - & $\mathrm{E}$ & Spain & [94] \\
\hline 0900 Ziraat & $600-650$ & 134 & - & $15500-16000$ & $\mathrm{E}$ & Turkey & [97] \\
\hline $\begin{array}{l}\text { Montmorency(sour } \\
\text { cherry) }\end{array}$ & - & 954 & - & 6130 & S & USA & [42] \\
\hline
\end{tabular}

\section{Concluding Remarks and Perspectives}

This study compiles the temperature requirements of a total of 530 cultivars of eight Prunus ssp. Most of the data correspond to peach (204 cultivars), almond (106 cultivars), Japanese apricot (77 cultivars), European apricot (68 cultivars), and sweet cherry (49 cultivars) since there is little information available for European plum (nine cultivars), Japanese plum (16 cultivars), and sour cherry ( 1 cultivar) (Table 8$)$. These data represent a very small percentage of the existing commercial cultivars and, in addition, 84 out of 530 cultivars came from studies published more than 25 years ago (Table 8). Therefore, temperature requirements are only available for very current growing cultivars. To serve as a reference, more than 1500 stone fruit cultivars were registered in the European Union in the last 25 years, including 946 for peach, 320 for apricot, 132 for Japanese plum, and 130 for sweet cherry [99]. Two main methodologies, statistical and experimental, have been used to obtain the temperature requirements reported here. Chilling requirements were experimentally determined for all the cultivars of European and Japanese apricot and European and Japanese plum. In contrast, most of the data available for cultivars of almond (153 statistical data vs. 56 experimental data) and peach (173 statistical data vs. 42 experimental data) were statistically determined (Table 8). 
Table 8. For each stone fruit crop, number of studies included in this work, number of cultivars with available data of chilling and/or heat requirements, and number of cultivars according to the methodology used.

\begin{tabular}{|c|c|c|c|c|c|c|}
\hline \multirow{2}{*}{\multicolumn{2}{|c|}{ Specie }} & \multirow{2}{*}{ Studies } & \multicolumn{2}{|c|}{ Cultivars } & \multicolumn{2}{|c|}{ Methodology } \\
\hline & & & Total $\mathbf{n}^{\circ}$ & $>25$ years old & Statistical & Experimental \\
\hline \multicolumn{2}{|c|}{ Almond } & 5 & 106 & 17 & 98 & 10 \\
\hline \multicolumn{2}{|c|}{ European apricot } & 15 & 68 & 23 & 0 & 69 \\
\hline \multicolumn{2}{|c|}{ Japanese apricot } & 3 & 77 & 0 & 0 & 77 \\
\hline \multirow{3}{*}{ Peach } & Flat peach & 1 & 7 & 0 & 1 & 0 \\
\hline & Nectarine & 3 & 25 & 0 & 3 & 0 \\
\hline & Peach & 18 & 172 & 1 & 7 & 11 \\
\hline \multicolumn{2}{|c|}{ European plum } & 1 & 9 & 9 & 0 & 9 \\
\hline \multicolumn{2}{|c|}{ Japanese plum } & 2 & 16 & 5 & 0 & 16 \\
\hline \multicolumn{2}{|c|}{ Sweet and Sour cherry } & 7 & 50 & 29 & 1 & 49 \\
\hline
\end{tabular}

Chilling quantification was performed according to different temperature models: 382 data were calculated with $\mathrm{CH}, 342$ with $\mathrm{CP}$ and 241 with $\mathrm{CP}$. Heat requirements are available for 328 out of 530 cultivars (Table 9). Among the different stone fruit crops, peach cultivars presented a wider range of chilling requirements (71-1390 CH, 5-1220 CU, and 1-1222 CP), while flat peach cultivars showed the least narrow range $(239-536 \mathrm{CH}, 354-861 \mathrm{CU}$, and 22.3-48.8 CP). Some almond cultivars showed the lowest chilling requirements, while some cultivars of European apricot, European plum, peach, and sweet cherry showed the highest chilling requirements. Japanese apricot cultivars showed lower heat requirements, while some peach cultivars showed the highest values (16493 GDH) (Table 9).

Table 9. For each stone fruit crop, the number of cultivars and range of both chilling and heat requirements according to the model used.

\begin{tabular}{|c|c|c|c|c|c|c|c|c|c|}
\hline \multirow{3}{*}{\multicolumn{2}{|c|}{ Specie }} & \multicolumn{6}{|c|}{ Chilling requirements } & \multirow{2}{*}{\multicolumn{2}{|c|}{$\begin{array}{c}\text { Heat requirements } \\
\text { GDH }\end{array}$}} \\
\hline & & \multicolumn{2}{|c|}{$\mathrm{CH}$} & \multicolumn{2}{|c|}{ CU } & \multicolumn{2}{|c|}{$\mathrm{CP}$} & & \\
\hline & & Data & Range & Data & Range & Data & Range & Data & Range \\
\hline \multicolumn{2}{|c|}{ Almond } & 73 & $8-713$ & 91 & $284-996$ & 62 & $3.4-55.4$ & 107 & $2862-10201$ \\
\hline \multicolumn{2}{|c|}{ Eur. apricot } & 78 & $171-1812$ & 88 & $274-1665$ & 31 & $29.8-78.9$ & 74 & $485-6729$ \\
\hline \multicolumn{2}{|c|}{ Jap. apricot } & 8 & $239-1148$ & 6 & $479-1323$ & 75 & $29-78.5$ & 75 & $822-2378$ \\
\hline \multirow{3}{*}{ Peach } & Flat peach & 7 & $239-536$ & 7 & $354-861$ & 7 & $22.3-48.5$ & 0 & - \\
\hline & Nectarine & 11 & $90-426$ & 24 & $45-1050$ & 9 & $9-47$ & 9 & $5853-9338$ \\
\hline & Peach & 132 & $71-1390$ & 93 & $5-1220$ & 34 & $1-122$ & 35 & $3476-16493$ \\
\hline \multicolumn{2}{|c|}{ Eur. plum } & 9 & $579-1323$ & 0 & - & 0 & - & 0 & - \\
\hline \multicolumn{2}{|c|}{ Jap. plum } & 16 & $118-685$ & 11 & $297-1053$ & 11 & $18-64$ & 11 & $5261-10034$ \\
\hline \multicolumn{2}{|c|}{ Sweet cherry } & 48 & $176-1100$ & 22 & $94-1559$ & 12 & $29-107$ & 17 & $3473-16000$ \\
\hline
\end{tabular}

The empirical methodology to determine dormancy by monitoring shoots in forcing conditions is the unique currently available, although it was designed more than 60 years ago [23]. This method has been applied to determine chilling requirements in early [32] and recent studies [31], but it has also been used to infer the dormant stage in research aimed at studying the physiology of dormancy $[6,54,59,100-102]$. The variability on the experimental designs among studies had often resulted in inconsistencies in the data obtained. This variability is noted in the frequency of shoot sampling along winter (e.g., weekly, every 10 days or every certain amount of chilling accumulated), but also in the environmental conditions of the growth chamber: temperature $\left(20^{\circ} \mathrm{C}, 25^{\circ} \mathrm{C}\right)$, temperature regime (constant or with day/night variation), and photoperiod. Subsequently, the evaluation of 
bud growth is performed after different periods in the growth chamber (a week, 10 days, 20 days), and it is based on analyzing vegetative [103] or flower buds [95]. Furthermore, several criteria are used to determine dormancy overcome. These can consist of significant increases in fresh [23] or dry weight [60], and/or phenological changes in bud phenology [24], which can result in an underestimation of the chilling requirements [98]. All these variations make this methodology easily adaptable to the characteristics of each fruit species and regional variations, but the results obtained under specific conditions should be taken with caution when applied to other regions or climates.

The statistical determination of dormancy is mainly based on two approaches. On one side, the correlation of winter temperatures with the flowering dates, which has been used to establish the chilling requirements of almond [26,33], apricot, peach, plum and sweet cherry [33] cultivars in Spain. On the other side, PLS analysis has been recently developed in a sweet cherry cultivar in Germany [34], and was subsequently applied to other temperate fruit crops as almond in Tunisia [35] and Spain [36], and apricot in China [27,104]. This methodology has reported interesting results on predicting phenology under future scenarios of global warming [19,104]. However, statistical analyses present low applicability on new cultivars released from breeding programs, since they are based on a long series of flowering dates records (more than 20 years).

Once the endo- and eco- dormancy periods have been established, specific temperature models are used to calculate the duration of each phase. Although these models were specially designed to quantify chilling or warm temperatures, they present numerous drawbacks. The Chilling Hours model is easy to understand and calculate and is commonly used by growers who often know the accumulated $\mathrm{CH}$ in their location, despite the lack of information about the requirements of their cultivars. This model does not fit perfectly with the behavior of trees, especially in mild and warm areas $[105,106]$. The Utah model attempts to be more accurate by weighting the temperature ranges. However, the fact that it establishes negative values for warm temperatures hampers its applicability in mild winter conditions. The different criteria for establishing the starting point to quantify temperatures, either an established date for dormancy starting (e.g., November $1^{\text {st }}$ ) [35] or the date with the maximum negative value [29], had resulted in high differences even for the same cultivar as occurs in almond [26,35,43] or European apricot $[45,48,50]$, making the comparison between studies difficult. The Dynamic model has been proposed as the best model available but also presents limitations on fitting the plant responses to chill [37]. It was designed as a process-based model, however, the physiological process behind is still unknown [30,40,72]. Finally, the Growing Degree Hours model allows quantifying forcing temperatures in a wide range of biological processes, such as phenological stages of annual crops or even insect growth [107]. The results of GDH quantification are highly variable between species, especially at different locations [34]). This could be due to both the model and the interaction between chill and heat accumulation [108-110].

In spite of both the methodologies to determine dormancy periods and the temperature models have numerous pitfalls, the available data of temperature requirements are useful to predict the adaptability of a particular cultivar to a certain area. However, very few of the cultivars currently grown have known temperature requirements. This means that, in most cases, the flowering period is the unique information available to assess the adaptation of a cultivar. Flowering periods are usually related to a reference cultivar (e.g., 'Burlat' in sweet cherry) [5] and successfully used for spring frost risk assessment and for pollination purposes, to predict flowering overlap between pollinating and pollinated cultivars. However, assessing the possible adaptation to an area based on relative flowering dates has many limitations, and temperature requirements offer a more reliable approach [111]. However, this review shows that information is not available for the most important cultivars nowadays, and, when available, they are usually imprecise estimations based only on flowering dates. Furthermore, temperature requirements are scarcely evaluated in most breeding programs, which could lead to an increasing lack of information in the coming years.

In conclusion, numerous improvements may be needed to obtain an accurate determination of the temperature requirements of stone fruit cultivars. The standardization of the experimental 
conditions would allow obtaining more robust and comparable data. However, the increasing number of new cultivars in most Prunus species and the expected reduction of winter chill due to global warming emphasize the necessity of a proper biological marker for dormancy. This would allow the analysis of samples collected directly from the field, without depending on external factors such as forcing conditions in the experimental approach or the availability of a large set of phenological data. Recent reports have revealed several processes as promising candidates for dormancy markers such as the expression of the DORMANCY-ASSOCIATED MAD-BOX (DAM) genes in peach [112], starch accumulation within the ovary primordia cell in sweet cherry [113], anther meiosis in apricot [114,115], and hormone regulation in sweet cherry [116]. Establishing the relationship between temperature records and a biological dormancy marker would lead to a process-based model that would allow direct determination of dormancy and a more accurate estimation of the temperature requirements of particular cultivars.

Author Contributions: J.R. and E.F. developed the conceptual framework of the manuscript; E.F., S.H., B.I.G., M.E.G., and J.R. wrote the paper. J.R. and E.F. corrected the final draft. All authors have read and agreed to the published version of the manuscript.

Funding: research was funded by Instituto Nacional de Investigación y Tecnología Agraria y Alimentaria (RFP2015-00015-00, RTA2017-00003-00); Gobierno de Aragón-European Social Fund, European Union (Grupo Consolidado A12_17R).

Conflicts of Interest: The authors declare no conflict of interest. The funders had no role in the design of the study; in the collection, analyses, or interpretation of data; in the writing of the manuscript, or in the decision to publish the results.

\section{References}

1. Fruit Breeding; Badenes, M.; Byrne, D. (Eds.) Springer: Boston, MA, USA, 2012.

2. FAOSTAT. Available online: http://www.fao.org/faostat/es/\#data/QC (accessed on 15 November 2019).

3. Perry, T.O. Dormancy of trees in winter. Science 1971, 171, 29-36. [CrossRef] [PubMed]

4. Fadón, E.; Herrero, M.; Rodrigo, J. Flower bud dormancy in Prunus species. In Advances in Plant Dormancy; Anderson, J.V., Ed.; Springer: Cham, Switzerland, 2015; pp. 123-135.

5. Guerra, M.E.; Rodrigo, J. Japanese plum pollination: A review. Sci. Hortic. 2015, 197, 674-686. [CrossRef]

6. Fadón, E.; Rodrigo, J. Unveiling winter dormancy through empirical experiments. Environ. Exp. Bot. 2018, 152, 28-36. [CrossRef]

7. Atkinson, C.J.; Brennan, R.M.; Jones, H.G. Declining chilling and its impact on temperate perennial crops. Environ. Exp. Bot. 2013, 91, 48-62. [CrossRef]

8. Luedeling, E.; Zhang, M.; Girvetz, E.H. Climatic changes lead to declining winter chill for fruit and nut trees in California during 1950-2099. PLoS ONE 2009, 4, e6166. [CrossRef]

9. Campoy, J.A.; Ruiz, D.; Egea, J. Dormancy in temperate fruit trees in a global warming context: A review. Sci. Hortic. 2011, 130, 357-372. [CrossRef]

10. Rohde, A.; Bhalerao, R.P. Plant dormancy in the perennial context. Trends Plant Sci. 2007, 12, $217-223$. [CrossRef]

11. Lang, G.A.; Early, J.D.; Martin, G.C.; Darnell, R.L. Endodormancy, paradormancy, and ecodormancyPhysiological terminology and classification for dormancy research. HortScience 1987, 22, 371-377.

12. Fadón, E.; Fernandez, E.; Behn, H.; Luedeling, E. A conceptual framework for winter dormancy in deciduous trees. Agronomy 2020, 10. [CrossRef]

13. Richardson, E.A.; Seeley, S.D.; Walker, D.R.; Anderson, J.L.; Ashcroft, G.L. Pheno-climatography of spring peach bud development. HortScience 1975, 10, 236-237.

14. Jansson, S.; Douglas, C.J. Populus: A model system for plant biology. Annu. Rev. Plant Biol. 2007, 58, 435-458. [CrossRef] [PubMed]

15. Castède, S.; Campoy, J.A.; García, J.Q.; Le Dantec, L.; Lafargue, M.; Barreneche, T.; Wenden, B.; Dirlewanger, E. Genetic determinism of phenological traits highly affected by climate change in Prunus avium: Flowering date dissected into chilling and heat requirements. New Phytol. 2014, 202, 703-715. [CrossRef] [PubMed]

16. Fadón, E.; Herrero, M.; Rodrigo, J. Flower development in sweet cherry framed in the BBCH scale. Sci. Hortic. 2015, 192, 141-147. [CrossRef] 
17. Considine, M.J.; Considine, J.A. On the language and physiology of dormancy and quiescence in plants. J. Exp. Bot. 2016, 67, 3189-3203. [CrossRef]

18. Benmoussa, H.; Ben Mimoun, M.; Ghrab, M.; Luedeling, E. Climate change threatens central Tunisian nut orchards. Int. J. Biometeorol. 2018, 62, 2245-2255. [CrossRef]

19. Benmoussa, H.; Luedeling, E.; Ghrab, M.; Ben Yahmed, J.; Ben Mimoun, M. Performance of pistachio (Pistacia vera L.) in warming Mediterranean orchards. Environ. Exp. Bot. 2017, 140, 76-85. [CrossRef]

20. Luedeling, E.; Girvetz, E.H.; Semenov, M.A.; Brown, P.H. Climate change affects winter chill for temperate fruit and nut trees. PLoS ONE 2011, 6, e20155. [CrossRef]

21. Guerra, M.E.; Guerrero, B.I.; Casadomet, C.; Rodrigo, J. Self- (in) compatibility, S-RNase allele identification, and selection of pollinizers in new Japanese plum-type cultivars. Sci. Hortic. 2020, 261, 109022. [CrossRef]

22. Guo, L.; Dai, J.; Ranjitkar, S.; Yu, H.; Xu, J.; Luedeling, E. Chilling and heat requirements for flowering in temperate fruit trees. Int. J. Biometeorol. 2014, 58, 1195-1206. [CrossRef]

23. Brown, D.S.; Kotob, F.A. Growth of flower buds of apricot, peach, and pear during the rest period. Proc. Am. Soc. Hortic. Sci. 1957, 69, 158-164.

24. Bennett, J.P. Temperature and bud rest period. Calif. Agric. 1949, 3, 9-12.

25. Ashcroft, G.L.; Richardson, E.A.; Seeley, S.D. A statistical method of determinig Chill Unit and Growing Degree Hour requirements for deciduous fruit trees. HortScience 1977, 12, 347-348.

26. Alonso, J.M.; Ansón, J.M.; Espiau, M.T.; Company, R.S. Determination of endodormancy break in almond flower buds by a correlation model using the average temperature of different day intervals and its application to the estimation of chill and heat requirements and blooming date. J. Am. Soc. Hortic. Sci. 2005, 130, 308-318. [CrossRef]

27. Luedeling, E.; Brown, P.H.; Girvetz, E.H.; Semenov, M.A.; Brown, P.H.; Guo, L.; Dai, J.; Ranjitkar, S.; Yu, H.; $\mathrm{Xu}$, J.; et al. Statistical identification of chilling and heat requirements for apricot flower buds in Beijing, China. Sci. Hortic. 2015, 55, 1-7.

28. Weinberger, J.H. Chilling requirements of peach varieties. Proc. Am. Soc. Hortic. Sci. 1950, 56, $122-128$.

29. Richardson, E.A.; Seeley, S.D.; Walker, D.R. A model for estimating the completion of rest for "Redhaven" and "Elberta" peach trees. HortScience 1974, 9, 331-332.

30. Fishman, S.; Erez, A.; Couvillon, G.A. The temperature dependence of dormancy breaking in plants: Mathematical analysis of at two-step model involving a cooperative transition. J. Theor. Biol. 1987, 124, 473-483. [CrossRef]

31. Ruiz, D.; Egea, J.; Salazar, J.A.; Campoy, J.A. Chilling and heat requirements of Japanese plum cultivars for flowering. Sci. Hortic. 2018, 242, 164-169. [CrossRef]

32. Tabuenca, M.C.; Herrero, J. Influencia de la temperatura en la época de floración de frutales. An. Estac. Exp. Aula Dei 1966, 8, 115-153.

33. Tabuenca, M.C.; Mut, M.; Herrero, J. The effect of temperature on flowering date in almond varieties. An. Estac. Exp. Aula Dei 1972, 11, 378-395.

34. Luedeling, E.; Kunz, A.; Blanke, M.M. Identification of chilling and heat requirements of cherry trees a statistical approach. Int. J. Biometeorol. 2013, 57, 679-689. [CrossRef]

35. Benmoussa, H.; Ben Mimoun, M.; Ghrab, M.; Luedeling, E. Chilling and heat requirements for local and foreign almond (Prunus dulcis Mill.) cultivars in a warm Mediterranean location over 30 years of observation. Agric. For. Meteorol. 2017, 239, 34-46. [CrossRef]

36. Díez-Palet, I.; Funes, I.; Savé, R.; Biel, C.; de Herralde, F.; Miarnau, X.; Vargas, F.; Àvila, G.; Carbó, J.; Aranda, X. Blooming under Mediterranean climate: Estimating cultivar-specific chill and heat requirements of almond and apple trees using a statistical approach. Agronomy 2019, 9, 760. [CrossRef]

37. Luedeling, E.; Brown, P.H. A global analysis of the comparability of winter chill models for fruit and nut trees. Int. J. Biometeorol. 2011, 55, 411-421. [CrossRef] [PubMed]

38. Couvillon, G.A.; Erez, A. Effect of level and duration of high temperatures on test in the peach. J. Am. Soc. Hortic. Sci. 1985, 110, 579-581.

39. Erez, A.; Couvillon, G.A.; Hendershott, C.H. Quantitative chilling enhancement and negation in peach buds by high temperatures in a daily cycle. J. Am. Soc. Hortic. Sci. 1979, 104, 536-540.

40. Erez, A.; Couvillon, G.A. Characterization of the moderate temperature effect on peach bud rest. J. Am. Soc. Hortic. Sci. 1987, 112, 667-680. 
41. de Reaumur, R.A.F. Observations du thermomètre, faites á Paris pendant l'année 1735, comparées avec celles ui ont été faites sous la ligne, á l'isle de France, á Alger et quelques unes de nos isles de l’Amérique. Mem. Acad. Sci. Paris 1735.

42. Anderson, J.L.; Richardson, E.A.; Kesner, C.D. Validation of Chill Unit and flower bud phenology models for "Montmorency"sour cherry. Acta Hortic. 1986, 184, 71-78. [CrossRef]

43. Egea, J.; Ortega, E.; Martínez-Gómez, P.; Dicenta, F. Chilling and heat requirements of almond cultivars for flowering. Environ. Exp. Bot. 2003, 50, 79-85. [CrossRef]

44. Hormaza, J.I.; Yamane, H.; Rodrigo, J. Apricot. In Fruits and Nuts. Genome Mapping and Molecular Breeding in Plants; Kole, C., Ed.; Springer: Berlin/Heidelberg, Germany, 2007.

45. Campoy, J.A.; Ruiz, D.; Allderman, L.; Cook, N.; Egea, J. The fulfilment of chilling requirements and the adaptation of apricot (Prunus armeniaca L.) in warm winter climates: An approach in Murcia (Spain) and the Western Cape (South Africa). Eur. J. Agron. 2012, 37, 43-55. [CrossRef]

46. Guerriero, R.; Monteleone, P.; Viti, R. Evaluation of end of dormancy in several apricot cultivars according to different methodological approaches. Acta Hortic. 2006, 701, 99-103. [CrossRef]

47. Zhebentyayeva, T.; Ledbetter, C.; Burgos, L.; Llacer, G. Apricot. In Fruit Breeding; Badenes, M.L., Byrne, D., Eds.; Springer: Boston, MA, USA, 2012.

48. Garcia, E.G.; Guerriero, R.; Monteleone, P. Apricot bud chilling and heat requirements in two different climatic areas: Murcia and the Tuscan Maremma. Acta Hortic. 1997, 488, 289-294. [CrossRef]

49. Viti, R.; Andreini, L.; Ruiz, D.; Egea, J.; Bartolini, S.; Iacona, C.; Campoy, J.A. Effect of climatic conditions on the overcoming of dormancy in apricot flower buds in two Mediterranean areas: Murcia (Spain) and Tuscany (Italy). Sci. Hortic. 2010, 124, 217-224. [CrossRef]

50. Ruiz, D.; Campoy, J.A.; Egea, J. Chilling and heat requirements of apricot cultivars for flowering. Environ. Exp. Bot. 2007, 61, 254-263. [CrossRef]

51. Ruml, M.; Milatović, D.; Đurović, D.; Zec, G.; Jokić, M.; Radović, M. Chilling and heat requirements for flowering in apricot cultivars. Acta Hortic. 2018, 1214, 15-18. [CrossRef]

52. Gao, Z.; Zhuang, W.; Wang, L.; Shao, J.; Luo, X.; Cai, B.; Zhang, Z. Evaluation of chilling and heat requirements in japanese apricot with three models. HortScience 2012, 47, 1826-1831. [CrossRef]

53. Zhuang, W.; Cai, B.; Gao, Z.; Zhang, Z. Determination of chilling and heat requirements of 69 Japanese apricot cultivars. Eur. J. Agron. 2016, 74, 68-74. [CrossRef]

54. Yamane, H.; Kashiwa, Y.; Kakehi, E.; Yonemori, K.; Mori, H.; Hayashi, K.; Iwamoto, K.; Tao, R.; Kataoka, I. Differential expression of dehydrin in flower buds of two Japanese apricot cultivars requiring different chilling requirements for bud break. Tree Physiol. 2006, 26, 1559-1563. [CrossRef] [PubMed]

55. Yamane, H.; Wada, M.; Honda, C.; Matsuura, T.; Ikeda, Y.; Hirayama, T.; Osako, Y.; Gao-Takai, M.; Kojima, M.; Sakakibara, H.; et al. Overexpression of Prunus DAM6 inhibits growth, represses bud break competency of dormant buds and delays bud outgrowth in apple plants. PLOS ONE 2019, 14, 1-24. [CrossRef]

56. Habu, T.; Yamane, H.; Sasaki, R.; Yano, K.; Fujii, H.; Shimizu, T.; Yamamoto, T.; Tao, R. Custom microarray analysis for transcript profiling of dormant vegetative buds of Japanese apricot during prolonged chilling exposure. J. Jpn. Soc. Hortic. Sci. 2014, 83, 1-16. [CrossRef]

57. Sasaki, R.; Yamane, H.; Ooka, T.; Jotatsu, H.; Kitamura, Y.; Akagi, T.; Tao, R. Functional and expressional analyses of PmDAM genes associated with endodormancy in Japanese apricot. Plant Physiol. 2011, 157, 485-497. [CrossRef] [PubMed]

58. Kitamura, Y.; Habu, T.; Yamane, H.; Nishiyama, S.; Kajita, K.; Sobue, T.; Kawai, T.; Numaguchi, K.; Nakazaki, T.; Kitajima, A.; et al. Identification of QTLs controlling chilling and heat requirements for dormancy release and bud break in Japanese apricot (Prunus mume). Tree Genet. Genomes 2018, 14, 33. [CrossRef]

59. Esumi, T.; Kitamura, Y.; Hagihara, C.; Yamane, H.; Tao, R. Identification of a TFL1 ortholog in Japanese apricot (Prunus mume Sieb. et Zucc.). Sci. Hortic. 2010, 125, 608-616. [CrossRef]

60. Tabuenca, M.C. Winter chilling requirements of apricot varieties. An. Estac. Exp. Aula Dei 1968, 9, 10-24.

61. Razavi, F.; Hajilou, J.; Tabatabaei, S.; Dadpour, M. Comparison of Chilling and heat requirement in some peach and apricot cultivars. Res. Plant Biol. 2011, 1, 40-47.

62. Valentini, N.; Ruffa, E.; Me, G.; Spanna, F.; Lovisetto, M. Chilling, thermal time and metabolic changes in five apricot varieties. Acta Hortic. 2006, 701, 147-150. [CrossRef] 
63. Tabuenca, M.C. Chilling requirements of apricot, peach and pear varieties. An. Estac. Exp. Aula Dei 1964, 7, 113-132.

64. Tabuenca, M.C. Duración del periodo de reposo a distintas temp y evaluación de las necesidades de frío en albaricoquero y almendro. An. Estac. Exp. Aula Dei 1979, 11, 325-329.

65. Andreini, L.; Viti, R.; Bartolini, S.; Ruiz, D.; Egea, J.; Campoy, J.A. The relationship between xylem differentiation and dormancy evolution in apricot flower buds (Prunus armeniaca L.): The influence of environmental conditions in two Mediterranean areas. Trees Struct. Funct. 2012, 26, 919-928. [CrossRef]

66. Valentini, N.; Me, G.; Spanna, F.; Lovisetto, M. Chilling and heat requirement in apricot and peach varieties. Acta Hortic. 2004, 636, 199-203. [CrossRef]

67. Bailey, C.H.; Cowgill, W.; Hough, L.F. Estimate of chilling requirements of apricot selections. Acta Hortic. 1977, 85, 184-189. [CrossRef]

68. Barbosa, W.; Chagas, E.A.; Pommer, C.V.; Pio, R. Advances in low-chilling peach breeding at Instituto Agronômico, São Paulo State, Brazil. Acta Hortic. 2010, 872, 147-150. [CrossRef]

69. Pensoa, G.; Citadin, I.; Scariotto, S.; Magalhães dos Santos, C.; Junior, A.; Bruckner, C.; Rodrigo, J. Development of peach flower buds under low winter chilling conditions. Agronomy. (In press).

70. Hancock, J.F.; Scorza, R.; Lobos, G.A. Peaches. In Temperate Fruit Crop Breeding: Germplasm to Genomics; Hancock, J.F., Ed.; Springer: New York, NY, USA, 2008; pp. 265-298.

71. Erez, A.; Couvillon, G.A.; Hendershott, C.H. The effect of cycle length on chilling negation by high temperatures in dormant peach leaf buds. J. Am. Soc. Hortic. Sci. 1979, 104, 573-576.

72. Erez, A.; Fishman, S.; Linsley-Noakes, G.C.; Allan, P. The dynamic model for rest completion in peach buds. Acta Hortic. 1990, 165-174. [CrossRef]

73. Li, Z.; Reighard, G.L.; Abbott, A.G.; Bielenberg, D.G. Dormancy-associated MADS genes from the EVG locus of peach [Prunus persica (L.) Batsch] have distinct seasonal and photoperiodic expression patterns. J. Exp. Bot. 2009, 60, 3521-3530. [CrossRef]

74. Rodriguez-A, J.; Sherman, W.B.; Scorza, R.; Wisniewski, M.; Okie, W.R. “Evergreen” peach, its inheritance and dormant behavior. J. Am. Soc. Hortic. Sci. 1994, 119, 789-792. [CrossRef]

75. Navarro, A.C.; Gazquez, A.G.; Montiel, F.G.; Soto, M.L.; Cos, J. Estimación de las necesidades de frío de variedades de melocotón de forma plana (paraguayos). In Proceedings of the XIV Congreso Nacional De Ciencias Hortícolas, Orihuela, Spain, 3-5 June 2015.

76. Maulión, E.; Valentini, G.H.; Kovalevski, L.; Prunello, M.; Monti, L.L.; Daorden, M.E.; Quaglino, M.; Cervigni, G.D.L. Comparison of methods for estimation of chilling and heat requirements of nectarine and peach genotypes for flowering. Sci. Hortic. 2014, 177, 112-117. [CrossRef]

77. Gariglio, N.F.; Mendow, M.; Weber, M.E.; Favaro, M.A.; González-Rossia, D.E.; Pilatti, R.A. Phenology and reproductive traits of peaches and nectarines in Central-East Argentina. Sci. Agric. 2009, 66, 757-763. [CrossRef]

78. Kwon, J.H.; Jun, J.H.; Nam, E.Y.; Chung, K.H.; Hong, S.S.; Yoon, I.K.; Yun, S.K.; Kwack, Y.B. Profiling diversity and comparison of Eastern and Western cultivars of Prunus persica based on phenotypic traits. Euphytica 2015, 206, 401-415. [CrossRef]

79. Sawamura, Y.; Suesada, Y.; Sugiura, T.; Yaegaki, H. Chilling requirements and blooming dates of leading peach cultivars and a promising early maturing peach selection, Momo Tsukuba 127. Hortic. J. 2017, 86, 426-436. [CrossRef]

80. Leida, C.; Romeu, J.F.; García-Brunton, J.; Ríos, G.; Badenes, M.L. Gene expression analysis of chilling requirements for flower bud break in peach. Plant Breed. 2012, 131, 329-334. [CrossRef]

81. Milech, C.; Dini, M.; Scariotto, S.; Santos, J.; Herter, F.; Raseira, M. Chilling requirement of ten peach cultivars estimated by different models. J. Exp. Agric. Int. 2018, 20, 1-9. [CrossRef]

82. Citadin, I.; Raseira, M.C.B.; Herter, F.G.; Baptista Da Silva, J. Heat requirement for blooming and leafing in peach. HortScience 2001, 36, 305-307. [CrossRef]

83. Chun, J.A.; Kang, K.; Kim, D.; Han, H.H.; Son, I.C. Prediction of full blooming dates of five peach cultivars (Prunus persica) using temperature-based models. Sci. Hortic. 2017, 220, 250-258. [CrossRef]

84. Li, Y.; Fang, W.C.; Zhu, G.R.; Cao, K.; Chen, C.W.; Wang, X.W.; Wang, L.R. Accumulated chilling hours during endodormancy impact blooming and fruit shape development in peach (Prunus persica L.). J. Integr. Agric. 2016, 15, 1267-1274. [CrossRef] 
85. Nishimoto, N.; Fujisaki, M. Chilling requirement of buds of some deciduous fruits grown in Southern Japan and the means to break dormancy. Acta Hortic. 1995, 153-160. [CrossRef]

86. Okie, W.R.; Blackburn, B. Increasing chilling reduces heat requirement for floral budbreak in peach. HortScience 2011, 46, 245-252. [CrossRef]

87. Ghrab, M.; Ben Mimoun, M.; Masmoudi, M.M.; Ben Mechlia, N. Chilling trends in a warm production area and their impact on flowering and fruiting of peach trees. Sci. Hortic. 2014, 178, 87-94. [CrossRef]

88. Mounzer, O.H.; Conejero, W.; Nicola, E.; Abrisqueta, I.; Tapia, L.M.; Vera, J.; Abrisqueta, J.M.; Ruiz-sa, M.C. Growth pattern and phenological stages of early-maturing peach trees under a mediterranean climate. HortScience 2008, 43, 1813-1818. [CrossRef]

89. Carrillo-Navarro, A.; Guevara-Gazquez, A.; García-Montiel, F.; López-Ortiz, D.; Fuentes-Denia, A.; López-Soto, M.B.; Caballero-Hernández, C.M.; Ruiz-García, L.; Cos-Terrer, J. Caracterización fenotípica y molecular de variedades del programa de mejora de melocotonero del IMIDA. In Proceedings of the IX Congreso De Mejora Genética De Plantas, Murcia, Spain, 18-20 September 2018.

90. Gariglio, N.; González Rossia, D.E.; Mendow, M.; Reig, C.; Agusti, M. Effect of artificial chilling on the depth of endodormancy and vegetative and flower budbreak of peach and nectarine cultivars using excised shoots. Sci. Hortic. 2006, 108, 371-377. [CrossRef]

91. Tabuenca, M.C. Winter chilling requirements of plum varieties. An. Estac. Exp. Aula Dei 1967, 8, 383-391.

92. Torrecillas, A.; Corell, M.; Galindo, A.; Pérez-López, D.; Memmi, H.; Rodríguez, P.; Cruz, Z.N.; Centeno, A.; Intrigliolo, D.S.; Moriana, A. Agronomical effects of deficit irrigation in apricot, peach, and plum trees. In Water scarcity and sustainable agriculture in semiarid environment; García-Tejero, I.F., Durán-Zuazo, V.H., Eds.; Elsevier-Academic Press: Cambridge, MA, USA, 2018; pp. 87-109. ISBN 9780128131640.

93. Gharbi, O.; Wünsch, A.; Rodrigo, J. Characterization of accessions of "Reine Claude Verte" plum using Prunus SRR and phenotypic traits. Sci. Hortic. 2014, 169, 57-65. [CrossRef]

94. Tabuenca, M.C. Winter chilling requierements of cherry varieties. An. Estac. Exp. Aula Dei 1983, 15, 661-667.

95. Fadón, E.; Rodrigo, J.; Herrero, M. Is there a specific stage to rest? Morphological changes in flower primordia in relation to endodormancy in sweet cherry (Prunus avium L.). Trees Struct. Funct. 2018, 32, 1583-1594. [CrossRef]

96. Alburquerque, N.; García-Montiel, F.; Carrillo, A.; Burgos, L. Chilling and heat requirements of sweet cherry cultivars and the relationship between altitude and the probability of satisfying the chill requirements. Environ. Exp. Bot. 2008, 64, 162-170. [CrossRef]

97. Kuden, A.B.; Imrak, B.; Bayazit, S.; Çömlekçioğlu, S.; Küden, A. Chilling requirements of cherries grown under subtropical conditions of Adana. Middle E. J. Sci. Res. 2012, 12, 1497-1501.

98. Campoy, J.A.; Darbyshire, R.; Dirlewanger, E.; Quero-garcía, J. Yield potential definition of the chilling requirement reveals likely underestimation of the risk of climate change on winter chill accumulation. Int. J. Biometeorol. 2019, 63, 183-192. [CrossRef]

99. Community Plant Variety Office (CPVO). Available online: https://europa.eu/european-union/about-eu/ agencies/cpvo_en (accessed on 15 November 2019).

100. Fadón, E.; Rodrigo, J. Combining histochemical staining and image analysis to quantify starch in the ovary primordia of sweet cherry during winter dormancy. J. Vis. Exp. 2019, 145, e58524. [CrossRef]

101. Fadón, E.; Herrero, M.; Rodrigo, J. Flower bud development and winter dormancy in sweet cherry (Prunus avium L.). Acta Hortic. 2019, 1231, 1-6. [CrossRef]

102. Fadón, E.; Herrero, M.; Rodrigo, J. Anther and pollen development in sweet cherry (Prunus avium L.) in relation to winter dormancy. Protoplasma 2019, 256, 733-744. [CrossRef] [PubMed]

103. Fernandez, E.; Cuneo, I.F.; Luedeling, E.; Alvarado, L.; Farias, D.; Saa, S. Starch and hexoses concentrations as physiological markers in dormancy progression of sweet cherry twigs. Trees Struct. Funct. 2019, 33, 1187-1201. [CrossRef]

104. Guo, L.; Dai, J.; Wang, M.; Xu, J.; Luedeling, E. Responses of spring phenology in temperate zone trees to climate warming: A case study of apricot flowering in China. Agric. For. Meteorol. 2015, 201, 1-7. [CrossRef]

105. Dennis, F.G. Problems in standardizing methods for evaluating the chilling requirements for the breaking of dormancy in buds of woody plants. HortScience 2003, 38, 347-350. [CrossRef]

106. Dennis, F.G. Dormancy-What we know (and Don't Know). HortScience 1994, 29, 1249-1255. [CrossRef]

107. Wilson, L.T.; Barnett, W.W. Degree-days: An aid in crop and pest management. Calif. Agric. 1983, 37, 4-7. 
108. Harrington, C.A.; Gould, P.J.; St.Clair, J.B. Modeling the effects of winter environment on dormancy release of Douglas-fir. For. Ecol. Manag. 2010, 259, 798-808. [CrossRef]

109. Pope, K.S.; Da Silva, D.; Brown, P.H.; DeJong, T.M. A biologically based approach to modeling spring phenology in temperate deciduous trees. Agric. For. Meteorol. 2014, 198-199, 15-23. [CrossRef]

110. Fernandez, E.; Luedeling, E.; Behrend, D.; Van de Vliet, S.; Kunz, A.; Fadón, E. Mild water stress in summer appears to affect dormancy in flower buds of apple advancing next spring phenology. Agronomy 2020, 10, 274. [CrossRef]

111. Herrera, S.; Lora, J.; Hormaza, J.; Rodrigo, J. Pollination management in stone fruit crops. In Production Technology of Stone Fruits; Ahmad Mir, S., Ahmad Shah, M., Maqbool Mir, M., Eds.; Springer-Verlag: Heidelberg, Germany, 2020.

112. Falavigna, V.d.S.; Guitton, B.; Costes, E.; Andrés, F. I want to (Bud) break free: The potential role of DAM and SVP-like genes in regulating dormancy cycle in temperate fruit trees. Front. Plant Sci. 2019, 9, 1-17. [CrossRef] [PubMed]

113. Fadón, E.; Herrero, M.; Rodrigo, J. Dormant flower buds actively accumulate starch over winter in sweet cherry. Front. Plant Sci. 2018, 9, 171. [CrossRef] [PubMed]

114. Julian, C.; Herrero, M.; Rodrigo, J. Anther meiosis time is related to winter cold temperatures in apricot (Prunus armeniaca L.). Environ. Exp. Bot. 2014, 100, 20-25. [CrossRef]

115. Julian, C.; Rodrigo, J.; Herrero, M. Stamen development and winter dormancy in apricot (Prunus armeniaca). Ann. Bot. 2011, 108, 617-625. [CrossRef]

116. Vimont, N.; Schwarzenberg, A.; Domijan, M.; Beauvieux, R.; Arkoun, M.; Yvin, J.-C.; Cortijo, S.; Wigge, P.A.; Dirlewanger, E.; Wenden, B. Hormonal balance finely tunes dormancy status in sweet cherry flower buds. BioRxiv 2019, 423871.

(C) 2020 by the authors. Licensee MDPI, Basel, Switzerland. This article is an open access article distributed under the terms and conditions of the Creative Commons Attribution (CC BY) license (http://creativecommons.org/licenses/by/4.0/). 